\title{
A Multicriteria Selection Framework for Wireless Communication Infrastructure with Interval-Valued Pythagorean Fuzzy Assessment
}

\author{
Shanshan Qiu, ${ }^{1}$ Dan $\mathrm{Fu}^{2}$, and Xiaofang Deng $\mathbb{D}^{1,3}$ \\ ${ }^{1}$ Software School, Jiangxi Normal University, Nanchang 330027, China \\ ${ }^{2}$ Foreign Languages College, Jiangxi Normal University, Nanchang 330022, China \\ ${ }^{3}$ School of Information and Communication Engineering, Guangzhou Maritime University, Guangzhou 510725, China \\ Correspondence should be addressed to Xiaofang Deng; dxf@jxnu.edu.cn
}

Received 5 March 2021; Revised 15 June 2021; Accepted 22 July 2021; Published 15 August 2021

Academic Editor: Wei Quan

Copyright ( 2021 Shanshan Qiu et al. This is an open access article distributed under the Creative Commons Attribution License, which permits unrestricted use, distribution, and reproduction in any medium, provided the original work is properly cited.

\begin{abstract}
In recent years, interval-valued Pythagorean fuzzy number is playing a more and more important role in decision management. It is a more effective and powerful tool to handle fuzzy information in decision problems. The multicriteria decision-making theory has been widely used in solving practical problems, such as the risk assessment of financial investment, engineering and construction, medical and health care, and information security. The main purpose of this paper is to apply a new interval-valued Pythagorean fuzzy decision-making method to practice and to analyze and solve the problem of wireless communication infrastructure. In this paper, a new interval-valued Pythagorean fuzzy ranking method, extending scope of application of the VIKOR method to interval-valued Pythagorean fuzzy set, is proposed. In order to adapt to actual needs, subjective and objective weights are combined to solve decision-making problems to enhance its practicality, validity, and effectiveness. An example of wireless communication infrastructure problem is provided to illustrate the rationality of this method and verify its advantages.
\end{abstract}

\section{Introduction}

In real life, people often cannot describe things accurately. Words like "almost," "probably," and other vague adjectives are often used to do the description. Therefore, fuzzy numbers are widely used, so they play an important role in decision-making. In 1965, Zadeh developed the mathematical expression of fuzzy things, called fuzzy set theory [1] (FS); it is widely used to deal with uncertain information in decision-making process. It is also an important method for fuzzy decision-making. FS expresses two dimensions: degree of certainty (degree of membership) and degree of uncertainty (degree of nonmembership). Yet, a neutral attitude that neither agrees nor opposes cannot be expressed by FS. Subsequently, Atanassov [2] extended fuzzy sets to intuitionistic fuzzy sets (IFS); he firstly took the hesitancy degree into consideration, which analyzes information from three dimensions: the degree of membership $u$, nonmembership $v$, and hesitancy $\pi$. IFS express support, opposition, and neu- trality at the same time to overcome the disadvantages of single membership degree of fuzzy sets. Thus, the reliability of decision information is greatly improved. However, there still are cases that cannot be handled by using intuitionistic fuzzy sets, such as $u+v>1$. To solve this problem, Yager [3] introduced the concept of Pythagorean fuzzy set, stipulating that the sum of the square of membership and nonmembership of Pythagorean fuzzy set is less than or equal to 1 . For example, if we describe a problem as follows: the definite metrics (membership degree) is 0.6 , indefinite metrics (nonmembership) is 0.8 , because $0.6+0.8>1$, which is beyond the representation of intuitionistic fuzzy sets, but the problem can be well described by Pythagoras fuzzy set. Therefore, Pythagorean fuzzy set extended its expression range greatly on the basis of intuitionistic fuzzy set. It helped fuzzy decision-making to solve more practical problems and enhanced its practicability greatly. With the further study of practical problems, there is also uncertainty among membership index. In 2016, Zhang [4] raised the concept of interval- 
valued Pythagorean fuzzy number. This theory followed the earlier concept of interval-valued intuitionistic fuzzy sets and extended Interval-valued Intuitive Fuzzy Set Theory. Besides, it enriched the previous Pythagorean fuzzy set decision method. Hereafter, Garg [5] extended Pythagorean fuzzy set theory to interval Pythagorean fuzzy environment and introduces some aggregation operators related to interval-valued Pythagorean fuzzy sets. Furthermore, Garg [6] extended the TOPSIS method and proposed a score function to determine the relative closeness coefficient in the interval Pythagorean fuzzy environment. Chen [7] proposed a novel function of relative closeness and uses the closenessbased assignment model to determine the ranking of alternatives. Garg [8] provided new Pythagorean fuzzy exponential aggregation operators and methods to solve MCDM problem under the Pythagorean fuzzy environment. A novel concept of linguistic interval-valued Pythagorean fuzzy set (LIVPFS) is presented in [9] to solve more MAGDM problems. Moreover, Garg [10] developed a new neutral addition and scalar multiplication operations to solve the MAGDM problems. These methods make fuzzy decision-making more widely used. For example, when we say a watermelon is ripe, there is $60 \% \sim 80 \%$ probability that it is ripe and $40 \% \sim 50 \%$ probability that it is unripe. In this case, researchers use interval values to make a description, and the above situation can be easily expressed as $([0.6,0.8],[0.4,0.5])$.

In the process of decision-making, experts often need to choose the best one among candidates. Therefore, ranking method is very important in the decision-making process for multicriteria fuzzy decision-making. It seems that the previous Pythagorean fuzzy number ranking method is effective to solve the multicriteria decision-making problems. Yager [3] defined a set of Pythagorean natural order relationships to compare two fuzzy numbers through their membership and nonmembership. However, there are some Pythagorean fuzzy number pairs which cannot be compared by Yager [3]. To solve this problem, Zhang [11] came up with a strict order relation, which takes the hesitancy into consideration. However, for some Pythagorean fuzzy number pairs, the order relation cannot be clearly distinguished. Therefore, the researchers turned to ranking methods based on score functions and accuracy functions. Zhang and $\mathrm{Xu}$ [12] defined a score function for Pythagorean fuzzy numbers by using the square difference between membership and nonmembership. If the membership degree is the same as the nonmembership degree in a pair of Pythagorean fuzzy numbers, they will be considered as no difference by [12], which is inconsistent with the actual situation. Similarly, the ranking method based on score function and accuracy function is also proposed by Ren et al. [13], which is sensitive to disturbance, and even very small disturbance on membership degree could probably result in completely different ranking results. Subsequently, $\mathrm{Ma}$ and $\mathrm{Xu}$ [14] also proposed similar ranking methods with [13], but the ranking method lacks robustness, and the score function and accuracy function of ranking methods in literature [13] and literature [14] did not take the influence of hesitation into account. This may lead to information loss to some extent. To overcome the defect in $[13,14]$, Peng and Dai [15] have defined a new ranking schema by incorporating hesitancy into the consideration of the score function. However, in solving practical problems, the result is different from that of other previous ranking methods. Zhang [4] proposed a new ranking algorithm based on relative closeness. Ranking score is obtained by calculating the distance between the positive and negative ideal solutions, but the ranking method in [4] cannot distinguish the two Pythagorean fuzzy numbers with equal membership degree and nonmembership degree. Considering that Zhang [16] gave an expression to describe the ratio index of Pythagorean fuzzy number, which can sort a set of Pythagorean fuzzy number pairs. However, this method depends too much on the set of Pythagorean fuzzy numbers. Once the number changed, it may lead to different results, lacking reliability and stability.

In view of multicriteria decision-making problem, researchers proposed many decision-making methods from different aspects. Since 1981, based on the thought of "the idea that the smaller the distance from positive ideal solution, or the larger the distance from the negative ideal solution, the better the fuzzy number it will be," Hwang and Yoon proposed the TOPSIS decision-making method [17]. Many researchers have studied and applied this method [12, 18-24]. To solve the problem of multicriteria decision-making with incomplete attribute information, the TODIM decisionmaking method of incomplete information was proposed in [25], considering that experts have reference dependence and loss avoidance behaviour. Later, many researchers have enriched the method [13,26-28]. Taking the maximum group utility value and the minimum individual regret value into account, the VIKOR method was proposed by Opricovic, in 1998. It is one of the most effective tools in multicriteria decision evaluation and helpful to compare the relationship between individual and group. Over the past 20 years, VIKOR has been continuously extended and successfully applied to various fields [29-34]. This paper also cites VIKOR methods to determine objective weights in multicriteria decisionmaking problems.

The theory of multicriteria decision-making is widely used in solving practical problems, such as the risk assessment of financial investment [35-37], engineering and construction [38], medical and health care $[39,40]$, information security [20], and other areas [41, 42]. Based on the development of $5 \mathrm{G}$ technology, this paper did some research on the new situation and problems in the construction and protection of wireless communication infrastructure, focusing on the location of $5 \mathrm{G}$ base stations and aiming at the balance of coverage, capacity, cost, and quality of current wireless networks. The proposed options are evaluated by fuzzy decisionmaking method to provide a better scheme for experts. They are also conducive to the rapid promotion and the use of $5 \mathrm{G}$ technology.

The contributions of this paper can be illustrated as follows.

(1) By considering the relationship between membership degree, nonmembership degree, and hesitancy degree, this paper proposed a new ranking method 
for interval-valued Pythagorean fuzzy number based on information reliability

(2) To solve the construction and protection of wireless communication infrastructure problem, this paper puts forward a decision-making schema which combines subjective and objective weights to reduce the strong influence of subjectivity on the rationality of decision-making

(3) The proposed method extends VIKOR method to interval-valued Pythagorean fuzzy environment. Based on this compromised VIKOR method, the proposed method makes an objective balance between the maximum group utility value and the minimum individual regret value according to the objective data and solves the $5 \mathrm{G}$ base station selection

The rest of this article is divided into six sections. Section 2 provides a brief introduction to fuzzy sets and some related theories. Section 3 analyzes and summarizes the previous ranking methods of Pythagorean fuzzy numbers and interval-valued Pythagorean fuzzy numbers. Section 4 introduces the interval-valued Pythagorean fuzzy number ranking method and the multicriteria decision method based on the ranking rule. Section 5 uses a real case to elaborate the working process of the proposed decision-making method. Section 6 compares the ranking method and decision method proposed in this paper with the previous research results in detail. Finally, in Section 7, we perform sensitivity analysis of the adjustment parameters involved in the proposed decision method.

\section{Preliminaries}

In this part, we will briefly introduce some basic definitions, properties, and distance formulas related to interval-valued Pythagorean fuzzy sets and then introduce an aggregation operator.

\subsection{Pythagorean Fuzzy Set}

Definition 1 (see [3]). Let $X$ be a nonempty domain, and the Pythagorean fuzzy set $P$ on $X$ is represented as follows.

$$
P=\left\{\left\langle x,\left(\mu_{P}(x), v_{P}(x)\right)\right\rangle \mid x \in X\right\},
$$

where $\mu_{P}(x)$ and $v_{P}(x)$ are the membership and nonmembership functions of element $x$ for set $P$, respectively, $\mu_{P}(x) \in[0,1]$ and $v_{P}(x) \in[0,1]$ and $0 \leq\left(\mu_{P}(x)\right)^{2}+\left(v_{P}(x)\right)^{2}$ $\leq 1$.

The following distance evaluation of two Pythagorean fuzzy numbers can be obtained.

$d\left(p_{1}, p_{2}\right)=\frac{1}{2}\left(\left|\left(\mu_{p_{1}}\right)^{2}-\left(\mu_{p_{2}}\right)^{2}\right|+\left|\left(v_{p_{1}}\right)^{2}-\left(v_{p_{2}}\right)^{2}\right|+\left|\left(\pi_{p_{1}}\right)^{2}-\left(\pi_{p_{2}}\right)^{2}\right|\right)$.

Based on the Pythagorean fuzzy set theory, the researchers make a thorough discussion on the interval- valued Pythagorean fuzzy set theory. The following is a brief introduction to the basic definition, properties, and distance formula of the interval-valued Pythagorean fuzzy set.

\subsection{Interval-Valued Pythagorean Fuzzy Sets}

Definition 2 (see [4]). Let the $X$ be a nonnull domain, and the interval-valued Pythagorean fuzzy set on the $X$ is $\tilde{P}$, represented as follows.

$$
\tilde{P}=\left\{<x,\left(\left[\mu_{\tilde{P}}^{L}(x), \mu_{\tilde{P}}^{U}(x)\right],\left[v_{\tilde{P}}^{L}(x), v_{\tilde{P}}^{U}(x)\right]\right)>\mid x \in X\right\},
$$

where $\left[\mu_{\tilde{P}}^{L}(x), \mu_{\tilde{P}}^{U}(x)\right]$ is the element $x$ for the membership range of the set, $\left[v_{\tilde{P}}^{L}(x), v_{\tilde{p}}^{U}(x)\right]$ is the element $x$ for the set of nonmembership value range, $0 \leq \mu_{\tilde{P}}^{L}(x)<\mu_{\tilde{P}}^{U}(x) \leq 1$ and $0 \leq$ $v_{\tilde{P}}^{L}(x)<v_{\tilde{P}}^{U}(x) \leq 1$ and $0 \leq\left(\mu_{\tilde{P}}^{U}(x)\right)^{2}+\left(v_{\tilde{P}}^{U}(x)\right)^{2} \leq 1$.

The distance evaluation of two interval-valued Pythagorean fuzzy numbers can be formulized as follows.

$$
\begin{aligned}
d\left(p_{1}, p_{2}\right)= & \frac{1}{4}\left(\left|\left(\mu_{p_{1}}^{L}\right)^{p}-\left(\mu_{p_{2}}^{L}\right)^{p}\right|+\left|\left(\mu_{p_{1}}^{U}\right)^{p}-\left(\mu_{p_{2}}^{U}\right)^{p}\right|\right. \\
& +\left|\left(v_{p_{1}}^{L}\right)^{p}-\left(v_{p_{2}}^{L}\right)^{p}\right|+\left|\left(v_{p_{1}}^{U}\right)^{p}-\left(v_{p_{2}}^{U}\right)^{p}\right| \\
& \left.+\left|\left(\pi_{p_{1}}^{L}\right)^{p}-\left(\pi_{p_{2}}^{L}\right)^{p}\right|+\left|\left(\pi_{p_{1}}^{U}\right)^{p}-\left(\pi_{p_{2}}^{U}\right)^{p}\right|\right) .
\end{aligned}
$$

2.3. Aggregation Operator. Because of the complexity of decision-making problem, there are a lot of interference factors in the decision-making process when experts judge the superiority of the candidates. Therefore, the aggregation operator for interval-valued Pythagorean fuzzy set is also very important. Here, the traditional geometric operator will be employed by the proposed ranking method in the paper.

Definition 3 (see [43]). Let the $z_{j}=\left\langle\left[\mu_{j L}, \mu_{j U}\right],\left[\mu v_{j L}, v_{j U}\right]\right\rangle$ $(j=1,2, \cdots, n)$ be the interval-valued Pythagorean fuzzy set. By weighted assembly operator, the matrix is $D$ based on the optional scheme and expressed as follows.

$$
\begin{aligned}
g_{n}= & g_{\omega}^{z_{n}}\left(z_{1}, z_{2}, \cdots, z_{j}\right) \\
= & \left\langle\left[1-\prod_{j=1}^{m}\left(1-\mu_{j L}\right)^{\omega^{j}}, 1-\prod_{j=1}^{m}\left(1-\mu_{j U}\right)^{\omega^{j}}\right],\right. \\
& \left.\cdot\left[\prod_{j=1}^{m} v_{j L} \omega^{j}, \prod_{j=1}^{m} v_{j U} \omega^{j}\right]\right\rangle .
\end{aligned}
$$

\section{Previous Ranking Methods}

This section introduced some proposed ranking methods, which contains some natural sequence relationship, knowledge measurement, and information reliability and some other ranking methods of Pythagorean fuzzy numbers and interval-valued Pythagorean fuzzy numbers, and the shortcomings of these methods are analyzed as well. 
3.1. Natural Sequence Relationship. Yager [1] defines a set of Pythagorean fuzzy number natural order relations. For a set of fuzzy number $P_{i}=\left(\mu_{i}, v_{i}\right)$, if $\mu_{1} \geq \mu_{2}$, and $v_{1} \leq v_{2}$, then $P_{1} \geq P_{2}$, which means $P_{1}$ is bigger than or indifferent to $P_{2}$, we get the representation which $P_{1}$ takes precedence over the $P_{2}$. By comparing the membership degree of two fuzzy numbers and the nonmembership degree, this method obtains the size relation. According to the definition in [1], there are some Pythagorean fuzzy number pairs that cannot be compared.

For example, there are two Pythagorean fuzzy numbers $P_{1}$ and $P_{2}$, where $P_{1}=(0.8,0.4)$ and $P_{2}=(0.7,0.3)$. The comparison shows that $\mu_{1} \geq \mu_{2}$ and $v_{1} \geq v_{2}$, we can not make a judgment.

To make up for the defect of Yager's method in [1], Zhang [11] puts forward strict order relationship and considers the hesitation degree.

For a set of fuzzy numbers $P_{i}=\left(\mu_{i}, v_{i}\right), \pi_{i}^{2}=1-\mu_{i}^{2}-v_{i}^{2}$, if $\mu_{1} \geq \mu_{2}, v_{1} \leq \nu_{2}$, and $\pi_{1} \leq \pi_{2}$, and there is a $P_{1} \geq P_{2}$, which means the priority of $P_{1}$ is higher than that of $P_{2}$. However, strict order relationships cannot distinguish certain Pythagorean fuzzy numbers.

For example, there are two Pythagorean fuzzy numbers $P_{1}$ and $P_{2}$, where $P_{1}=(0.8,0.4)$ and $P_{2}=(0.7,0.5)$. It can be seen that $\mu_{1} \geq \mu_{2}, \nu_{1} \leq \nu_{2}$, but $\pi_{1} \geq \pi_{2}$, so it can not be judged by this method.

3.2. Ranking Methods for Pythagorean Fuzzy Numbers. Zhang and $\mathrm{Xu}$ [12] defined a score function that, let $P_{1}=$ $\left(\mu_{1}, v_{1}\right)$ and $P_{2}=\left(\mu_{2}, v_{2}\right)$ be two Pythagorean fuzzy numbers, and the function they proposed as follows.

$$
S_{\text {zhang }}(\tilde{p})=\mu^{2}-v^{2} \text {, }
$$

The following ranking rules are defined.

$$
\begin{aligned}
& \text { if } S_{\text {zhang }}\left(P_{1}\right)<S_{\text {zhang }}\left(P_{2}\right), \text { then } \quad P_{1} \prec P_{2} \text {, } \\
& \text { if } S_{\text {zhang }}\left(P_{1}\right)=S_{\text {zhang }}\left(P_{2}\right), \quad \text { then } \quad P_{1} \sim P_{2} \text {, }
\end{aligned}
$$

where $\prec$ means smaller than and $\sim$ means indifferent to.

According to above methods, we find that the ranking method cannot be compared in some cases. For Pythagorean fuzzy numbers $P_{1}=(0.8,0.4)$ and $P_{2}=(0.7,0.1)$, the value can be computed as follows.

$$
\begin{aligned}
& S_{\text {zhang }}\left(P_{1}\right)=0.8^{2}-0.4^{2}=0.48 ; \\
& S_{\text {zhang }}\left(P_{2}\right)=0.7^{2}-0.1^{2}=0.48 .
\end{aligned}
$$

Since $S_{\text {zhang }}\left(P_{1}\right)=S_{\text {zhang }}\left(P_{2}\right)$, we got $P_{1} \sim P_{2}$. But it is obvious that the $P_{1}$ and $P_{2}$ are two different fuzzy numbers, so the judgment method is unreasonable.

To solve the above problems, the method [13] is motivated by [12], and it can be described as follows.

$$
S_{\text {peng }}(\tilde{p})=\mu^{2}+v^{2}
$$

Moreover, following ranking rules are defined.

$$
\begin{aligned}
& \text { if } S_{\text {zhang }}\left(P_{1}\right)<S_{\text {zhang }}\left(P_{2}\right), \text { then } \quad P_{1} \prec P_{2} \text {, } \\
& \text { if } S_{\text {zhang }}\left(P_{1}\right)=S_{\text {zhang }}\left(P_{2}\right), \\
& \text { if } S_{\text {peng }}\left(P_{1}\right)<S_{\text {peng }}\left(P_{2}\right), \text { then } \quad P_{1} \prec P_{2} \text {, } \\
& \text { if } S_{\text {peng }}\left(P_{1}\right)=S_{\text {peng }}\left(P_{2}\right), \text { then } \quad P_{1} \sim P_{2} .
\end{aligned}
$$

The method [13] has improved and overcome the shortcomings of [12]; it seems more reasonable, but in fact, there are still some problems. For example, existed two Pythagorean fuzzy numbers $P_{1}$ and $P_{2}, P_{1}=(0.8,0.4), P_{2}=(0.7,0.1)$, from formula (5), $S_{\text {zhang }}\left(P_{1}\right)=S_{\text {zhang }}\left(P_{2}\right)$, we cannot make the right judgment; then, we reuse formula (7) $S_{\text {peng }}\left(P_{1}\right)=08^{2}$ $+0.4^{2}=0.80>S_{\text {peng }}\left(P_{2}\right)=0.50=0.7^{2}+0.1^{2}$, that is to say $P_{1}>P_{2}$. When we consider making a small change in $P_{1}$ as a $P_{1}^{\prime}$, and set $P_{1}^{\prime}$ to $P_{1}^{\prime}=(0.7999,0.4)$, and the difference between the original $P_{1}$ of the membership value is only 0.0001 . This time we get $S_{\text {zhang }}\left(P_{1}^{\prime}\right)<S_{\text {zhang }}\left(P_{2}\right)$, which is contrary to the original result. Therefore, we concluded that this ranking method is less robust and sensitive to perturbations of small values, and the ranking method is unstable.

Zhang [4] focuses on distance measurement, and the ranking result is obtained according to the distance between Pythagorean number and ideal solution.

They defined $A(1,0)$ and $B(0,1)$ representation point is $A$ a positive ideal solution point; the point is $B$ a negative ideal solution point, and the distance between the positive and negative ideal solution is expressed as $d(P, A)$ and $d(P, B)$, respectively, and

$$
d(P, A)=\frac{1}{2}\left(\left|\left(\mu_{P}\right)^{2}-\left(\mu_{A}\right)^{2}\right|+\left|\left(\nu_{P}\right)^{2}-\left(\nu_{A}\right)^{2}\right|+\left|\left(\pi_{P}\right)^{2}-\left(\pi_{A}\right)^{2}\right|\right) .
$$

There is a set of Pythagorean fuzzy numbers $\tilde{P}=(\tilde{\mu}, \tilde{v})$; by measuring the distance, Zhang [4] defines the following scoring functions.

$$
c(\tilde{p})=\frac{d(\tilde{P}, B)}{d(\tilde{P}, A)+d(\tilde{P}, B)}=\frac{1-v^{2}}{2-\mu^{2}-v^{2}} .
$$

The ranking method is described as follows.

$$
\begin{array}{lll}
\text { if } c\left(P_{1}\right)<c\left(P_{2}\right), & \text { then } & P_{1} \prec P_{2}, \\
\text { if } c\left(P_{1}\right)=c\left(P_{2}\right), & \text { then } & P_{1} \sim P_{2} .
\end{array}
$$

For example, there are two Pythagorean fuzzy numbers $P_{1}=(0.7,0.7)$ and $P_{2}=(0.5,0.5)$. The calculation shows that $c\left(P_{1}\right)=c\left(P_{2}\right)=0.5$, then $P_{1} \sim P_{2}$. It can be obviously concluded that $P_{1}$ and $P_{2}$ are two different fuzzy numbers, so the judgment method is unreasonable.

3.3. Knowledge Measurement and Information Reliability. The correlation ranking method about comprehensive knowledge measure and information reliability was proposed 


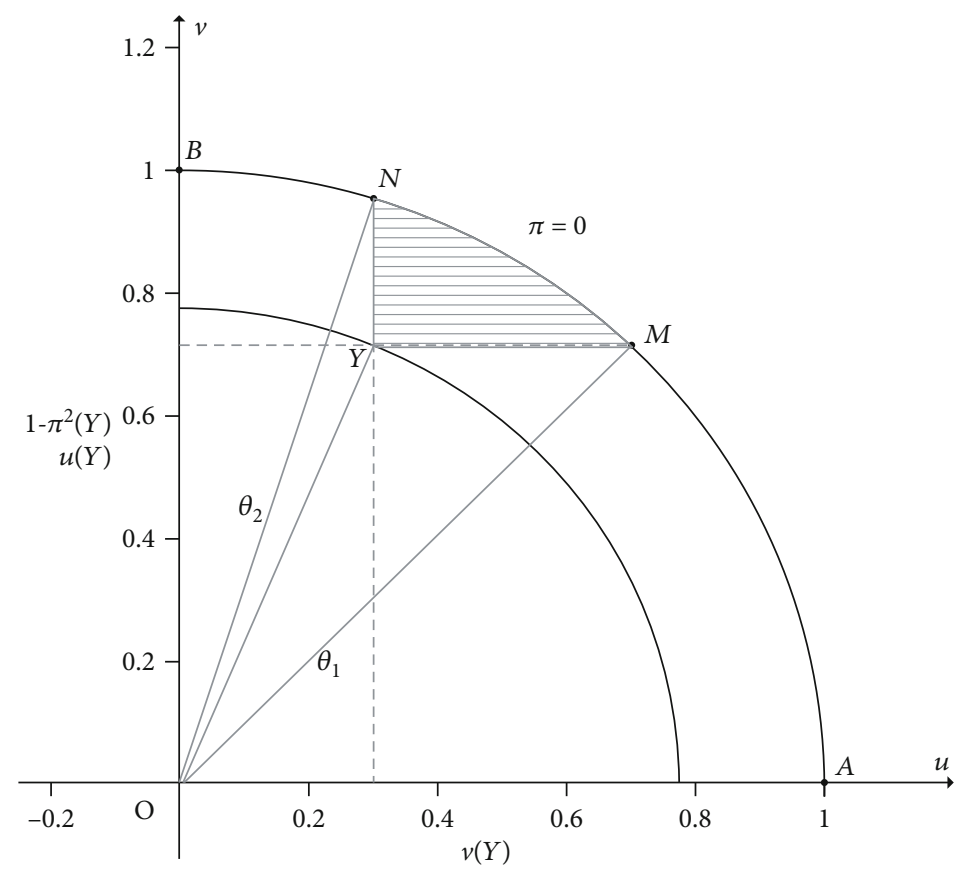

Figure 1: Schematic illustration of Pythagorean fuzzy number geometry.

in [44]. If there is a Pythagorean fuzzy number $Y(\mu, \nu, \pi)$, set a preference factor $\delta(0 \leq \delta<1 / 2)$. Based on the theory of distance measurement from positive and negative ideal solutions, they introduce two Pythagorean fuzzy numbers $A(1,0,0)$ and $B(0,1,0)$ and use formulas $d\left(Y, P_{\text {near }}\right)$ and $d\left(Y, P_{\text {far }}\right)$ to express a closer or longer distance between $Y$ and $A$ and $B$.

The expression of the defined knowledge measure function are as follows:

$$
K(Y)=\delta\left[1-\frac{1}{2}\left(\frac{d\left(Y, P_{\text {near }}\right)}{d\left(Y, P_{\text {far }}\right)}+\pi^{2}\right)\right]+(1-\delta) d(Y, O)
$$

where $\delta$ is a preference parameter.

Generally, if there is no extra preference relationship, $\delta=1 / 2$ means that expert treats the two components equally.

As shown in Figure 1, the arc $A B$ represents the point of $\mu^{2}+v^{2}=1$, and the hesitation value of any point on the arc is 0 . At this point, we call the Pythagorean fuzzy number 1 . The point in the sector area $O A B$ can be described as $0<\mu^{2}+v^{2}$ $<1$. The Pythagorean fuzzy number information represented by these points is more or less hesitant. There is a Pythagorean fuzzy number $Y$ located in the regional $O A B$. The projection of the arc $A B$ in the $\mu$ direction and the $v$ direction is represented by points $M$ and $N$, respectively. The shaded area $M Y N$ can be used to indicate the distance between the point $Y$, and the $\operatorname{arc} M N$ is an uncertain degree of information $Y$. The smaller the shadow $M Y N$ area, the more reliable the information represented by the Pythagorean fuzzy number $Y$ [45].

$$
S(Y)=1-S_{M Y N}=1-\left(S_{M O N}-S_{\Delta M O Y}-S_{\triangle N O Y}\right)=1-\frac{1}{2} \pi^{2} .
$$

Definition 4 (see [45]). There is a set of Pythagorean fuzzy number $P_{i}=\left(\mu_{i}, v_{i}, \pi_{i}\right)(i=1,2)$. The ranking rules for knowledge measurement and information reliability are as follows.

$$
\begin{aligned}
& \text { if } K\left(P_{1}\right)<K\left(P_{2}\right), \text { then } P_{1} \prec P_{2}, \\
& \text { if } K\left(P_{1}\right)=K\left(P_{2}\right), \\
& \text { if } S\left(P_{1}\right)<S\left(P_{2}\right), \text { then } P_{1} \prec P_{2}, \\
& \text { if } S\left(P_{1}\right)=S\left(P_{2}\right), \quad \text { then } \quad P_{1} \sim P_{2} .
\end{aligned}
$$

This method can calculate the Pythagorean correlation ranking results well and completely, but it is impossible to distinguish the interval-valued Pythagorean fuzzy numbers. Therefore, we extend this method to the interval-valued Pythagorean fuzzy environment to expand the scope of practical application.

\subsection{Ranking Methods for Interval-Valued Pythagorean Fuzzy Numbers}

3.4.1. Closeness Index-Based Score Function. In order to sort two interval-valued Pythagorean fuzzy numbers, the corresponding method was proposed in [4] which makes judgement base on the distance of positive and negative ideal solutions; the main ideas of this method are as follows.

To define an interval-valued Pythagorean fuzzy number $\beta=P\left(\left[\mu_{\beta}^{L}, \mu_{\beta}^{U}\right],\left[v_{\beta}^{L}, v_{\beta}^{U}\right]\right)$ and $o^{+}, o^{-}$represents its positive and negative ideal solution, which $o^{+}=P([1,1],[0,0])$ and 
$o^{-}=P([0,0],[1,1])$. Then, the function of $\beta$ is defined as follows.

$$
\begin{aligned}
\zeta(\beta) & =\frac{d\left(\beta, O^{-}\right)}{d\left(\beta, O^{-}\right)+d\left(\beta, O^{+}\right)} \\
& =\frac{2-\left(v_{\beta}^{L}\right)^{2}-\left(v_{\beta}^{U}\right)^{2}}{4-\left(\mu_{\beta}^{L}\right)^{2}-\left(\mu_{\beta}^{U}\right)^{2}-\left(v_{\beta}^{L}\right)^{2}-\left(v_{\beta}^{U}\right)^{2}} .
\end{aligned}
$$

The ranking rules can be described as follows.

$$
\begin{aligned}
& \text { if } \zeta\left(P_{1}\right)<\zeta\left(P_{2}\right), \quad \text { then } \quad P_{1} \prec P_{2}, \\
& \text { if } \zeta\left(P_{1}\right)=\zeta\left(P_{2}\right), \quad \text { then } \quad P_{1} \sim P_{2} .
\end{aligned}
$$

Example 1. There are two interval-valued Pythagorean fuzzy numbers $P_{1}$ and $P_{2} ; P_{1}=([0.5,0.6],[0.5,0.6])$, and $P_{2}=$ $([0.4,0.7],[0.4,0.7])$. The calculation shows that $\zeta\left(P_{1}\right)=$ $\zeta\left(P_{2}\right)=0.5$; we obtained that $P_{1} \sim P_{2}$. But, obviously, the fuzzy number $P_{1}$ is different from $P_{2}$, where the membership and nonmembership ranges are consistent; the Pythagorean fuzzy numbers cannot be compared.

And then, Garg [46] developed an improved score function for interval-valued Pythagorean fuzzy numbers, to define an interval-valued Pythagorean fuzzy number $P=([$ $\left.\left.\mu_{P}^{L}(x), \mu_{P}^{U}(x)\right],\left[\nu_{P}^{L}(x), v_{P}^{U}(x)\right]\right)$, which is denoted by $P=([a$, $b],[c, d])$. And they gave the score function of $P$ as follows.

$M(P)=\frac{\left(a^{2}-c^{2}\right)\left(1+\sqrt{1-a^{2}-c^{2}}\right)+\left(b^{2}-d^{2}\right)\left(1+\sqrt{1-b^{2}-d^{2}}\right)}{2}$,

where $M(P) \in[-1,1]$.

Based on the definition of $M(P)$, the ranking rules can be described as follows.

$$
\begin{array}{lll}
\text { if } M\left(P_{1}\right)<M\left(P_{2}\right), & \text { then } & P_{1} \prec P_{2}, \\
\text { if } M\left(P_{1}\right)=M\left(P_{2}\right), & \text { then } & P_{1} \sim P_{2} .
\end{array}
$$

Example 2. There are two interval-valued Pythagorean fuzzy numbers $P_{1}$ and $P_{2} ; P_{1}=([0,1],[0,0])$, and $P_{2}=([1,1],[0,0])$. The calculation shows that $M\left(P_{1}\right)=M\left(P_{2}\right)=0$; we obtained $P_{1} \sim P_{2}$. Obviously, $P_{1}$ and $P_{2}$ are different, but we have come to the conclusion that $P_{1}=P_{2}$. Therefore, Garg's method cannot distinguish $P_{1}$ and $P_{2}$.

\subsubsection{Novel Pearson-Like Correlation-Based TOPSIS Model.} Similarly, the Pearson-based correlation measure method and the interval-valued Pythagorean correlation coefficient method were proposed in [47] to solve the ranking problem of interval-valued Pythagorean fuzzy number.

Let $P_{i 1}$ and $P_{i 2}$ be two values in the interval-valued Pythagorean fuzzy matrix $D$. The interval-valued Pythagorean fuzzy correlation coefficient of $\gamma^{w} g\left(P_{i 1}, P_{i 2}\right)$ between $P_{i 1}$ and $P_{i 2}$ is defined as follows.

$$
\begin{aligned}
\gamma^{w}\left(P_{i 1}, P_{i 2}\right)= & \frac{1}{6}\left(r_{\mu}^{w-}\left(P_{i 1}, P_{i 2}\right)+r_{\mu}^{w+}\left(P_{i 1}, P_{i 2}\right)+r_{v}^{w-}\left(P_{i 1}, P_{i 2}\right)\right. \\
& \left.+r_{v}^{w+}\left(P_{i 1}, P_{i 2}\right)+r_{\pi}^{w-}\left(P_{i 1}, P_{i 2}\right)+r_{\pi}^{w+}\left(P_{i 1}, P_{i 2}\right)\right),
\end{aligned}
$$

which,

$$
\begin{aligned}
& r_{\mu}^{-}\left(P_{i 1}, P_{i 2}\right)=\frac{\sum_{j=1}^{n} w_{j}\left(\left(\mu_{i_{1}}^{-}\right)^{2}-\left(\bar{\mu}_{j}^{-}\right)^{2}\right) \cdot\left(\left(\mu_{i_{1}}^{-}\right)^{2}-\left(\bar{\mu}_{j}^{-}\right)^{2}\right)}{\sqrt{\sum_{j=1}^{n} w_{j}\left(\left(\mu_{i_{j}}^{-}\right)^{2}-\left(\bar{\mu}_{j}^{-}\right)^{2}\right)^{2}} \cdot \sqrt{\sum_{j=1}^{n} w_{j}\left(\left(\mu_{i_{j}}^{-}\right)^{2}-\left(\bar{\mu}_{j}^{-}\right)^{2}\right)^{2}}}, \\
& r_{\mu}^{+}\left(P_{i 1}, P_{i 2}\right)=\frac{\sum_{j=1}^{n} w_{j}\left(\left(\mu_{i_{1}}^{-}\right)^{2}-\left(\bar{\mu}_{j}^{+}\right)^{2}\right) \cdot\left(\left(\mu_{i_{i}}^{-}\right)^{2}-\left(\bar{\mu}_{j}^{+}\right)^{2}\right)}{\sqrt{\sum_{j=1}^{n} w_{j}\left(\left(\mu_{i_{i}}^{-}\right)^{2}-\left(\bar{\mu}_{j}^{+}\right)^{2}\right)^{2}} \cdot \sqrt{\sum_{j=1}^{n} w_{j}\left(\left(\mu_{i_{1}}^{-}\right)^{2}-\left(\bar{\mu}_{j}^{+}\right)^{2}\right)^{2}}} .
\end{aligned}
$$

On the basis of the TOPSIS method and the Pearson correlation coefficient, the interval-valued Pythagorean correlation closeness index $\mathrm{CI}(P)$ of interval-valued Pythagorean numbers can be defined as follows.

$$
\mathrm{CI}(P)=\eta \cdot \mathrm{CC}_{a}^{w}\left(P_{i}\right)+(1-\eta) \cdot \mathrm{CC}_{v}^{w}\left(P_{i}\right),
$$

where

$$
\begin{aligned}
\mathrm{CC}_{a}^{w}\left(P_{i}\right) & =\frac{1-\gamma^{w}\left(P_{i}, P_{\#}\right)}{2-\gamma^{w}\left(P_{i}, P_{\#}\right)-\gamma^{w}\left(P_{i}, P_{*}\right)}, \\
\mathrm{CC}_{v}^{w}\left(P_{i}\right) & =\frac{1+\gamma^{w}\left(P_{i}, P_{\#}\right)}{2+\gamma^{w}\left(P_{i}, P_{\#}\right)+\gamma^{w}\left(P_{i}, P_{*}\right)},
\end{aligned}
$$

where $P_{i}$ means interval-valued Pythagorean numbers of matrix $D, P_{*}$ means an characteristics of positive ideal solution, and $P_{\#}$ means a characteristics of negative ideal solution.

The larger the CI's value, the better the fuzzy number. The $\mathrm{CC}_{a}^{w}\left(P_{i}\right)$ and $\mathrm{CC}_{v}^{w}\left(P_{i}\right)$ are the interval-valued Pythagorean correlation coefficients based on approximation and avoidance attitude, and the parameter $\eta$ is the adjustment coefficient of approximation and avoidance, where $0<\eta<1$.

Example 3. For two sets of interval-valued Pythagorean fuzzy numbers $P_{1}$ and $P_{2}, p_{1}([0.2,0.3],[0.4,0.6])$ and $p_{2}([0.5,0.8]$, [ $0.4,0.6]$ ) are with same nonmembership degree. Our intuitive comparison of membership can be concluded quickly, but the results cannot be obtained by using the above method. Therefore, the method has some defects.

3.4.3. Accuracy Function and Distance Measures. In [5], Garg introduced an accuracy function of interval-valued Pythagorean fuzzy numbers. Let $P=\left(\left[\mu_{P}^{L}(x), \mu_{P}^{U}(x)\right],\left[v_{P}^{L}(x), v_{P}^{U}(x)\right]\right)$ which is denoted by $P=([a, b],[c, d])$. And there are three score functions as follows. 


$$
\begin{gathered}
S(P)=\frac{a^{2}+b^{2}-c^{2}-d^{2}}{2}, \\
H(P)=\frac{a^{2}+b^{2}+c^{2}+d^{2}}{2}, \\
M_{g}(P)=\frac{a^{2}-\sqrt{1-a^{2}-c^{2}}+b^{2}-\sqrt{1-b^{2}-d^{2}}}{2},
\end{gathered}
$$

where $S(P) \in[-1,1], H(P) \in[-1,1]$, and $M_{g}(P) \in[-1,1]$.

Let $P_{1}$ and $P_{2}$ be any two interval-valued Pythagorean fuzzy numbers. Then,

$$
\begin{gathered}
\text { if } S\left(P_{1}\right)<S\left(P_{2}\right), \quad \text { then } \quad P_{1}<P_{2}, \\
\text { if } S\left(P_{1}\right)=S\left(P_{2}\right), \quad \text { then } \quad P_{1} \sim P_{2}, \\
\text { if } H\left(P_{1}\right)<H\left(P_{2}\right), \text { then } \quad P_{1} \prec P_{2}, \\
\text { if } H\left(P_{1}\right)=H\left(P_{2}\right), \text { then } \quad P_{1} \sim P_{2}, \\
\text { if } M_{g}\left(P_{1}\right)<M_{g}\left(P_{2}\right), \quad \text { then } \quad P_{1} \prec P_{2}, \\
\text { if } M_{g}\left(P_{1}\right)=M_{g}\left(P_{2}\right), \quad \text { then } \quad P_{1} \sim P_{2} .
\end{gathered}
$$

Example 4. There are two interval-valued Pythagorean fuzzy numbers $P_{1}$ and $P_{2} ; P_{1}=([0.4,0.6],[0.6,0.8])$, and $P_{2}=([0.5$, $\sqrt{0.27}],[\sqrt{0.27}, \sqrt{0.73}])$. The calculation shows that $\mathrm{S}\left(P_{1}\right)=S\left(P_{2}\right)=-0.24, H\left(P_{1}\right)=H\left(P_{2}\right)=0.76$, and $M_{g}\left(P_{1}\right)$ $=M_{g}\left(P_{2}\right) \approx-0.0864$. We obtained $P_{1} \sim P_{2}$. Obviously, $P_{1}$ and $P_{2}$ are different, but we have come to the conclusion that $P_{1}=P_{2}$. Therefore, Garg's method cannot distinguish $P_{1}$ and $P_{2}$.

To overcome this problem, Garg [48] developed a different accuracy function. For an interval-valued Pythagorean fuzzy number $P=\left(\left[\mu_{P}^{L}(x), \mu_{P}^{U}(x)\right],\left[\nu_{P}^{L}(x), v_{P}^{U}(x)\right]\right)$ which is also denoted by $P=([a, b],[c, \mathrm{~d}])$, an improved accuracy function $K$ is defined by

$$
M_{\mathrm{Grag}}(P)=\frac{a^{2}+b^{2} \sqrt{1-a^{2}-c^{2}}+b^{2}+a^{2} \sqrt{1-b^{2}-d^{2}}}{2},
$$

where $M_{\mathrm{Grag}}(P) \in[-1,1]$.

Based on the function, the ranking rules can be described as follows.

$$
\begin{array}{lll}
\text { if } M_{\mathrm{Grag}}\left(P_{1}\right)<M_{\mathrm{Grag}}\left(P_{2}\right), & \text { then } & P_{1} \prec P_{2}, \\
\text { if } M_{\mathrm{Grag}}\left(P_{1}\right)=M_{\mathrm{Grag}}\left(P_{2}\right), & \text { then } & P_{1} \sim P_{2} .
\end{array}
$$

Example 5. There are two interval-valued Pythagorean fuzzy numbers $P_{1}$ and $P_{2} ; P_{1}=([0,1],[0,0])$, and $P_{2}=([1,1],[0,0])$. The calculation shows that $M_{\mathrm{Grag}}\left(P_{1}\right)=M_{\mathrm{Grag}}\left(P_{2}\right)=0$. We obtained $P_{1} \sim P_{2}$. Obviously, $P_{1}$ and $P_{2}$ are different, but we have come to the conclusion that $P_{1}=P_{2}$. Therefore, Garg's method cannot distinguish $P_{1}$ and $P_{2}$. So it still has some disadvantage of those accuracy functions.
Kumar et al. [49] put forward the interval-valued Pythagorean fuzzy ranking method about accuracy function and distance measure; the method can be illustrated as follows.

Set of interval-valued Pythagorean fuzzy number $p=$ $\left(\left[\underline{\mu}_{p}(u), \bar{\mu}_{p}(u)\right],\left[\underline{v}_{p}(u), \bar{v}_{p}(u)\right]\right)$, a transformation of the expression of $P$, recorded as $p=\left(\left[\underline{r}_{p}, \bar{r}_{p}\right],\left[\underline{d}_{p}, \bar{d}_{p}\right]\right)$, of which $\left[\underline{r}_{p}, \bar{r}_{p}\right]$ represents the lower and upper strength of interval-valued Pythagorean fuzzy number; $\left[\underline{d}_{p}, \bar{d}_{p}\right]$ represents the lower and upper strength directions of intervalvalued Pythagorean fuzzy numbers. The relationship between $p=\left(\left[\underline{\mu}_{p}(u), \bar{\mu}_{p}(u)\right],\left[\underline{v}_{p}(u), \bar{v}_{p}(u)\right]\right)$ and $\left[\underline{r}_{p}, \bar{r}_{p}\right],\left[\underline{d}_{p}\right.$, $\left.\bar{d}_{p}\right]$ is as follows.

$$
\begin{gathered}
\underline{\mu}_{p}(u)=\underline{r}_{p} \cos \left(\underline{\theta}_{p}\right), \underline{v}_{p}(u)=\underline{r}_{p} \sin \left(\underline{\theta}_{p}\right), \\
\bar{\mu}_{p}(u)=\bar{r}_{p} \cos \left(\bar{\theta}_{p}\right), \bar{v}_{p}(u)=\bar{r}_{p} \sin \left(\bar{\theta}_{p}\right), \\
\underline{d}_{p}=\left(1-\frac{2 \underline{\theta}_{p}}{\pi}, 1-\frac{2 \bar{\theta}_{p}}{\pi}\right) .
\end{gathered}
$$

According to the above relations, in order to solve the problem of comparison between Pythagorean fuzzy numbers during the period, an extended accuracy function is proposed. For each Pythagorean fuzzy number $p=\left(\left[\underline{\mu}_{p}(u)\right.\right.$, $\left.\left.\bar{\mu}_{p}(u)\right],\left[\underline{v}_{p}(u), \bar{v}_{p}(u)\right]\right)$, the following accuracy functions are defined as follows.

$$
T(p)=\frac{2\left(\underline{r}_{p} \underline{d}_{p}+\bar{r}_{p} \bar{d}_{p}\right)-\left(\underline{r}_{p}+\bar{r}_{p}\right)+2}{4},
$$

where

$$
\begin{aligned}
& \underline{r}_{p}=\sqrt{\left(\underline{\mu}_{p}(u)\right)^{2}+\left(\underline{v}_{p}(u)\right)^{2}}, \\
& \bar{r}_{p}=\sqrt{\left(\bar{\mu}_{p}(u)\right)^{2}+\left(\bar{v}_{p}(u)\right)^{2}},
\end{aligned}
$$

$$
\underline{d}_{p}=\left(1-\frac{2 \underline{\theta}_{p}}{\pi}, 1-\frac{2 \bar{\theta}_{p}}{\pi}\right) .
$$

And define the comparison rules as follows.

$$
\begin{aligned}
& \text { if } T\left(p_{1}\right)<T\left(p_{2}\right) \text { then } p_{1} \prec p_{2} \text {, } \\
& \text { if } T\left(p_{1}\right)>T\left(p_{2}\right) \text { then } p_{1}>p_{2} \text {, } \\
& \text { if } T\left(p_{1}\right)=T\left(p_{2}\right) \text { then } p_{1} \sim p_{2} \text {. }
\end{aligned}
$$




\section{A New Ranking Method of Interval-Valued Pythagorean Fuzzy Numbers Based on Information Reliability}

From what we have discussed in the previous section, there are still some problems with the ordering of two intervals of Pythagorean fuzzy numbers.

(1) If the membership and nonmembership of the interval Pythagorean are equal (equivalent to the Pythagorean fuzzy number), it may not be possible to distinguish between such two intervals with the previous method

(2) For two different intervals of Pythagorean fuzzy numbers, when we use previous method to compare the size, sometimes the two fuzzy numbers are the same, so the size cannot be compared

(3) The use of previous methods may sometimes go against popular perception and lead to wrong conclusions

In order to solve these problems, we propose a new interval Pythagorean fuzzy sorting function.

4.1. Ranking Function. In practical applications, the membership degree and nonmembership degree of a candidate may only be expressed by interval number, which cannot be determined as a certain number. Based on the above considerations, we extend the ranking method to interval-valued Pythagorean fuzzy numbers to expand the available range of the ranking method to solve more practical problems.

Based on the idea of the method proposed by the [50], we make the assumptions as follows.

Two sets of interval-valued Pythagorean fuzzy number $P=\left(\left[\mu_{p}^{L}, \mu_{p}^{U}\right],\left[v_{p}^{L}, v_{p}^{U}\right]\right), P^{\prime}=\left(\left[\mu_{p^{\prime}}^{L}, \mu_{p^{\prime}}^{U}\right],\left[\nu_{p^{\prime}}^{L}, v_{p^{\prime}}^{U}\right]\right)$, the value distribution is shown in Figure 2; the shadow $S 1$ indicates the degree of information uncertainty in Pythagorean fuzzy $P$.

Inspired by the ranking thought of literature [50], an interval Pythagorean fuzzy number $P=\left\{\left[\mu_{p}^{L}, \mu_{p}^{U}\right],\left[v_{p}^{L}, v_{p}^{U}\right]\right\}$ can be expressed in the rectangular coordinate axis as following four points. If we express it in the right axis, we can get the following points $P_{1}\left(\mu_{p}^{L}, v_{p}^{L}\right), P_{2}\left(\mu_{p}^{L}, v_{p}^{U}\right), P_{3}\left(\mu_{p}^{U}, v_{p}^{L}\right)$, and $P_{4}\left(\mu_{p}^{U}, v_{p}^{U}\right)$.

We call $P_{4}$ the most credible point. The hesitancy degree of point $P_{4}$ can be expressed in the area $y_{3} p_{4} y_{4}$ [50] and recorded as $S_{1}$. Suppose there are two interval Pythagorean fuzzy numbers $P_{A 4}$ and $P_{B 4}$, when these two points take the optimal values $P_{A 4}$ and $P_{B 4}$, we can calculate their information reliability. The larger $S_{1}$ is, the more hesitant the interval-valued Pythagorean fuzzy number $P$ is, and the less credible the interval-valued Pythagorean fuzzy number $P$ is.

However, when two interval-valued Pythagorean fuzzy numbers have the same degree of hesitation, we may not be able to compare them. To achieve the preciseness of the ranking method, we propose to compare information reliability in $\mu$ direction and $v$ direction. Compared with the two points
$P_{1}\left(\mu_{p}^{L}, v_{p}^{L}\right)$ and $P_{3}\left(\mu_{p}^{U}, v_{p}^{L}\right)$, the $\mu$ values are taken as the two ends of the interval $\left[\mu_{p}^{L}, \mu_{p}^{U}\right]$, respectively. The degree of hesitation in point $P_{1}$ is expressed in area $S_{y_{2} p_{1} y_{1}}$, and the degree of hesitation in point $P_{3}$ is expressed in area $S_{y_{3} p_{3} y_{1}}$. Then, the difference between those two areas is the hesitation of interval-valued Pythagorean fuzzy numbers in the $\mu$ direction and recorded as $S_{2}$, which, $S_{2}=S_{y_{2} p_{1} p_{3} y_{3}}=S_{y_{2} p_{1} y_{1}}-$ $S_{y_{3} p_{3} y_{1}}$, and when the $S_{2}$ gets bigger, the hesitation degree of $P$ gets bigger, and the credibility degree gets lower.

Likewise, the interval-valued Pythagorean fuzzy number hesitation degree of $P$ in the $v$ direction is recorded as $S_{3}$, which $S_{3}=S_{y_{4} p_{1} p_{2} y_{1}}=S_{y_{2} p_{1} y_{1}}-S_{y_{2} p_{2} y_{4}}$, and when the $S_{3}$ gets bigger, the hesitation degree of $P$ gets bigger, and its credibility gets lower, and the less credible it is. Inspired by [49], $S_{1}$, $S_{2}$, and $S_{3}$ can be calculated as follows.

$$
\begin{gathered}
S 1=S_{y_{3} p_{4} y_{4}}=S_{y_{3} o y_{4}}-S_{y_{3} o p_{4}}-S_{y_{4} o p_{4}}=\frac{1}{2} \pi_{p_{4}}^{2}, \\
S_{2}=S_{y_{2} p_{1} p_{3} y_{3}}=S_{y_{2} p_{1} y_{1}}-S_{y_{3} p_{3} y_{1}} \\
=\left(S_{y_{2} o y_{1}}-S_{y_{2} o p_{1}}-S_{y_{1} o p_{1}}\right)-\left(S_{y_{3} o y_{1}}-S_{y_{3} o p_{1}}-S_{y_{3} o p_{1}}\right) \\
=\frac{1}{2} \pi_{p_{1}}^{2}-\frac{1}{2} \pi_{p_{3}}^{2}, \\
=\left(S_{y_{2} o y_{1}}-S_{y_{2} o p_{1}}-S_{y_{1} o p_{1}}\right)-\left(S_{y_{2} o y_{4}}-S_{y_{2} o p_{2}}-S_{y_{4} o p_{2}}\right) \\
=\frac{1}{2} \pi_{p_{1}}^{2}-\frac{1}{2} \pi_{p_{2}}^{2} .
\end{gathered}
$$

In order to compare interval-valued Pythagorean fuzzy numbers, we propose the following ranking methods.

$$
\begin{aligned}
& \text { if } S_{1}\left(p_{1}\right) \leq S_{1}\left(p_{2}\right) \quad \text { then } \quad P_{1} \geq P_{2}, \\
& \quad \text { if } S_{1}\left(p_{1}\right)=S_{1}\left(p_{2}\right), \\
& \text { if } S_{2}\left(p_{1}\right) \leq S_{2}\left(p_{2}\right) \text { then } P_{1} \geq P_{2}, \\
& \quad \text { if } S_{2}\left(p_{1}\right)=S_{2}\left(p_{2}\right), \\
& \text { if } S_{3}\left(p_{1}\right) \leq S_{3}\left(p_{2}\right) \text { then } P_{1} \geq P_{2}, \\
& \text { if } S_{3}\left(p_{1}\right)=S_{3}\left(p_{2}\right) \text { then } P_{1} \sim P_{2} .
\end{aligned}
$$

Apply the new ranking method proposed in this paper to the previous methods described above, and we can get that.

In Example $1, P_{1}=([0.5,0.6],[0.5,0.6])$, and $P_{2}=([$ $0.4,0.7],[0.4,0.7])$, we get $S_{1}\left(P_{1}\right)=0.14$, and $S_{1}\left(P_{2}\right)=0.1$. The rank of those two interval-valued Pythagorean fuzzy number is $P_{1 \prec} P_{2}$.

In Examples 2 and 5, $P_{1}=([0,1],[0,0])$, and $P_{2}=([1,1]$, $[0,0])$, we get $S_{1}\left(P_{1}\right)=S_{1}\left(P_{2}\right)=0, S_{2}\left(P_{1}\right)=0.5$, and $S_{2}\left(P_{2}\right)=$ 0 . The rank of those two interval-valued Pythagorean fuzzy number is $P_{1 \prec} P_{2}$. 


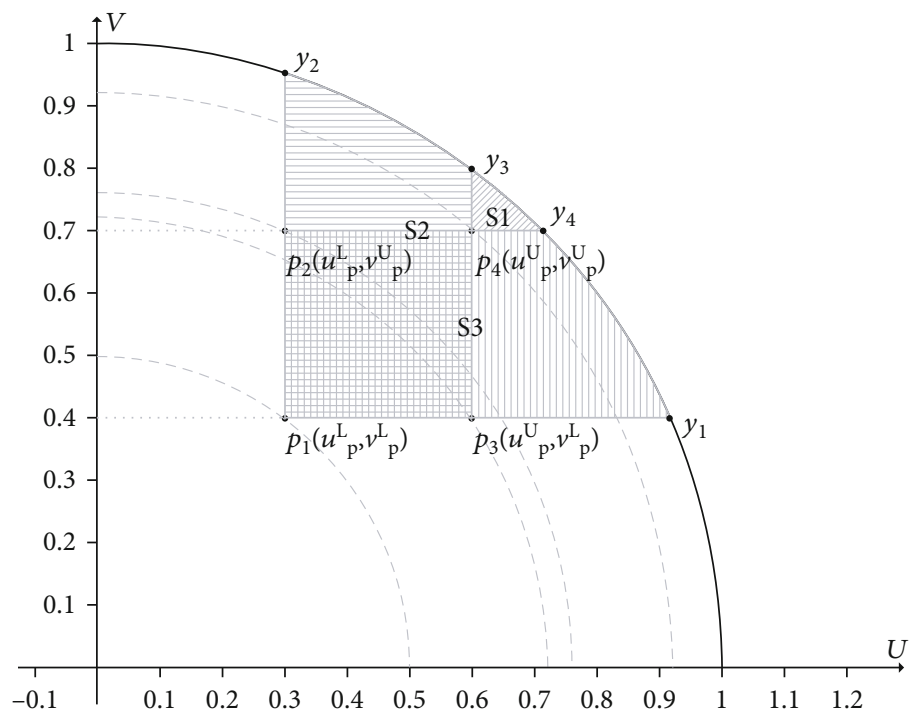

Figure 2: Schematic diagram of interval-valued Pythagorean fuzzy number.

In Example 3, $P_{1}=([0.2,0.3],[0.4,0.6])$, and $P_{2}=($ $0.5,0.8],[0.4,0.6])$, we get $S_{1}\left(P_{1}\right)=0.275$, and $S_{1}\left(P_{2}\right)=0$. The rank of those two interval-valued Pythagorean fuzzy number is $P_{1 \prec} P_{2}$.

In Example $4, P_{1}=([0.4,0.6],[0.6,0.8])$, and $P_{2}=(0.5$, $\sqrt{0.27}],[\sqrt{0.27}, \sqrt{0.73}])$, we get $S_{1}\left(P_{1}\right)=S_{1}\left(P_{2}\right)=0, S_{2}\left(P_{1}\right)$ $=0.1$, and $S_{2}\left(P_{2}\right)=0.01$. The rank of those two intervalvalued Pythagorean fuzzy number is $P_{1 \prec} P_{2}$.

There are some unreasonable problems in the previous ranking methods. We list the defects of these ranking methods as Table 1.

Obviously, we can compare the two interval-valued Pythagorean fuzzy sets with the same membership degree and nonmembership degree. We can also compare the Pythagorean fuzzy numbers between two intervals with the same nonmembership degree. Therefore, the proposed method can solve some problems that cannot be compared before, and this has practical significance.

4.2. Extended VIKOR Decision-Making Methods. Because the research of Pythagorean fuzzy decision-making is still in its infancy and the extension of Pythagorean fuzzy set has the advantage of information representation, it is of practical significance to find a decision-making method for Pythagorean fuzzy set to solve practical problems. VIKOR (Vlse Kriterijuska Optimizacija I Komoromisno Resenje) is a multiattribute decision-making method proposed by Opricovic [51] in 1998. By calculating the positive and negative ideal solutions of each option and combining the preferences of experts, the alternatives are sorted according to the evaluation value of the scheme and the proximity of the ideal scheme. Based on the maximum group utility value and the minimum individual regret value, this study summarizes the current decision methods of Pythagorean fuzzy numbers. It is considered that the extension of multicriteria compromise solution scheduling method (VIKOR) to Pythagorean fuzzy environment will help to solve more practical problems. VIKOR method is a compromise method, which sorts the finite decision scheme by maximizing group utility and minimizing individual regret.

4.2.1. Determination of Positive and Negative Ideal Solution. Set $\mathrm{A}$, as a collection of $m$ options, represents $A=\left\{A_{1}, A_{2}\right.$, $\left.\cdots, A_{m}\right\} ; C$ is a collection of $n$ evaluation criteria, which represents $C=\left\{C_{1}, C_{2}, \cdots, C_{n}\right\}$. The evaluation criteria $C$ is divided into two categories $C^{+}$and $C^{-}$, where $C^{+} \subset C, C^{-} \subset C, C^{+} \cap$ $C^{-}=\phi, C^{+}$indicates that the criteria were benefit-oriented, and $\mathrm{C}^{-}$indicates that the criteria is cost-based. To obtain a set of performance data $a_{i j}=\left(\left[\mu_{i j}^{L}, \mu_{i j}^{U}\right]\left[v_{i j}^{L}, v_{i j}^{U}\right]\right)(i=1,2, \cdots$, $m ; j=1,2, \cdots, n)$ for $m$ options of $n$ evaluation criteria in the form of interval-valued Pythagorean fuzzy numbers, of which $\left[\mu_{i j}^{L}, \mu_{i j}^{U}\right]$ is the membership value range, which $0 \leq \mu_{i j}^{L}<\mu_{i j}^{U} \leq 1,\left[v_{i j}^{L}, v_{i j}^{U}\right]$ is the value range of nonmembership, which $0 \leq v_{i j}^{L}<v_{i j}^{U} \leq 1 v_{i j}^{U^{2}}+u_{i j}^{U^{2}} \leq 1$.

Generally, we can obtain an interval-valued Pythagorean decision matrix $D$ as follows.

$$
\begin{aligned}
& C_{1} C_{2} \cdots C_{n}, \\
& D=\left(a_{i j}\right)_{m \times n}=\begin{array}{c}
A_{1} \\
A_{1} \\
\vdots \\
A_{1}
\end{array}\left[\begin{array}{cccc}
Z_{11} & Z_{21} & \cdots & Z_{n 1} \\
Z_{21} & Z_{22} & \cdots & Z_{n 2} \\
\vdots & \vdots & \ddots & \vdots \\
Z_{m 1} & Z_{m 2} & \cdots & Z_{m n}
\end{array}\right] .
\end{aligned}
$$

As for a Pythagorean fuzzy set, the larger value of $\mu$ and the smaller value of $v$, the bigger the value is. Likewise, interval numbers $([1,1],[0,0])$ and numbers $([0,0],[1,1])$ can be regarded as positive and negative ideal solutions of intervalvalued Pythagorean fuzzy sets. In terms of solving practical problems, it is sometimes unreasonable to simply set a fixed value as a positive and negative ideal solution. Therefore, we propose to calculate the corresponding positive and 
TABLE 1: Shortcoming of previous ranking methods.

\begin{tabular}{|c|c|c|}
\hline Method used & Score function & $\begin{array}{l}\text { Shortcoming (some fuzzy numbers cannot be } \\
\text { judged) }\end{array}$ \\
\hline Yager [1] & If $\mu_{1} \geq \mu_{2}$ and $v_{1} \leq v_{2}$, then $P_{1} \geq P_{2}$ & If $\mu_{1} \geq \mu_{2}$ and $v_{1} \geq v_{2}$ \\
\hline Zhang [11] & $\mu_{1} \geq \mu_{2}, v_{1} \leq v_{2}$, and $\pi_{1} \leq \pi_{2}$, then $P_{1} \geq P_{2}$ & If $\mu_{1} \geq \mu_{2}, v_{1} \leq v_{2}, \pi_{1} \geq \pi_{2}$ \\
\hline $\begin{array}{l}\text { Zhang and } \\
\mathrm{Xu}[12]\end{array}$ & $S_{\text {zhang }}(\tilde{p})=\mu^{2}-v^{2}$ & e.g., $P_{1}=(0.8,0.4)$, and $P_{2}=(0.7,0.1)$ \\
\hline Peng [13] & $S_{\text {peng }}(\tilde{p})=\mu^{2}+v^{2}$ & Robustness \\
\hline Zhang [4] & $\zeta(\beta)=\frac{d\left(\beta, O^{-}\right)}{d\left(\beta, O^{-}\right)+d\left(\beta, O^{+}\right)}=\frac{2-\left(v_{\beta}^{L}\right)^{2}-\left(v_{\beta}^{U}\right)^{2}}{4-\left(\mu_{\beta}^{L}\right)^{2}-\left(\mu_{\beta}^{U}\right)^{2}-\left(v_{\beta}^{L}\right)^{2}-\left(v_{\beta}^{U}\right)^{2}}$ & $\begin{aligned} \text { e.g., } P_{1}= & ([0.5,0.6],[0.5,0.6]), \text { and } P_{2}= \\
& ([0.4,0.7],[0.4,0.7])\end{aligned}$ \\
\hline Ho [47] & $\mathrm{CI}(P)=\eta \cdot \mathrm{CC}_{a}^{w}\left(P_{i}\right)+(1-\eta) \cdot \mathrm{CC}_{v}^{w}\left(P_{i}\right)$ & e.g., $p_{1}([0.2,0.3],[0.4,0.6])$, and $p_{2}([0.5,0.8],[0.4,0.6])$ \\
\hline Garg [5] & $\begin{array}{c}S(P)=\frac{a^{2}+b^{2}-c^{2}-d^{2}}{2} \\
H(P)=\frac{a^{2}+b^{2}+c^{2}+d^{2}}{2} \\
M_{g}(P)=\frac{a^{2}-\sqrt{1-a^{2}-c^{2}}+b^{2}-\sqrt{1-b^{2}-d^{2}}}{2}\end{array}$ & $\begin{array}{l}\text { e.g., } P_{1}=([0.4,0.6],[0.6,0.8]) \text {, and } P_{2}= \\
\qquad([0.5, \sqrt{0.27}],[\sqrt{0.27}, \sqrt{0.73}])\end{array}$ \\
\hline Garg [48] & $M_{\mathrm{Grag}}(P)=\frac{a^{2}+b^{2} \sqrt{1-a^{2}-c^{2}}+b^{2}+a^{2} \sqrt{1-b^{2}-d^{2}}}{2}$ & e.g., $P_{1}=([0,1],[0,0])$, and $P_{2}=([1,1],[0,0])$ \\
\hline Garg [46] & $M(P)=\frac{\left(a^{2}-c^{2}\right)\left(1+\sqrt{1-a^{2}-c^{2}}\right)+\left(b^{2}-d^{2}\right)\left(1+\sqrt{1-b^{2}-d^{2}}\right)}{2}$ & e.g., $P_{1}=([0,1],[0,0])$, and $P_{2}=([1,1],[0,0])$ \\
\hline
\end{tabular}

negative ideal solutions for each criteria according to the actual standard data values.

Let the positive ideal solution be expressed as $Z^{+}=$ $\left\{\left[\mu_{Z^{+}}^{L}, \mu_{Z^{+}}^{U}\right],\left[v_{Z^{+}}^{L}, v_{Z^{+}}^{U}\right]\right\}$. The standard data column is represented as $C_{n}=\left\{Z_{1 n}, Z_{2 n}, \cdots, Z_{m n}\right\}$, where $Z_{m n}=\left\{\left[\mu_{m n}^{L}, \mu_{m n}^{U}\right]\right.$, $\left.\left[v_{m n}^{L}, v_{m n}^{U}\right]\right\}$, According to the theory of positive and negative ideal solution, the maximum value of the upper and lower bounds of the membership degree in all schemes is taken as the upper and lower bounds of the membership degree of the positive ideal solution, and the minimum value of the upper and lower bounds of the nonmembership degree of the positive ideal solution is taken as the upper and lower bounds of the nonmembership degree of the positive ideal solution. In the same way, we hold the minimum of upper and lower bounds of membership as the upper and lower bounds of nonmembership degree of the positive ideal solution and hold the maximum of upper and lower bounds of nonmembership as the upper and lower bounds of nonmembership degree of the positive ideal solution. $Z^{+}$and $Z^{-}$are defined as follows.

$$
\begin{aligned}
Z^{+} & =\left\{\left[\max \mu_{\mathrm{mn}}^{L}, \max \mu_{\mathrm{mn}}^{U}\right],\left[\min v_{\mathrm{mn}}^{L}, \min v_{\mathrm{mn}}^{U}\right]\right\} \\
Z^{-} & =\left\{\left[\min \mu_{\mathrm{mn}}^{L}, \min \mu_{\mathrm{mn}}^{U}\right],\left[\max v_{\mathrm{mn}}^{L}, \max v_{\mathrm{mn}}^{U}\right]\right\} .
\end{aligned}
$$

4.2.2. Integrated Assessment Value. A conventional VIKOR method has the following rules.

$$
S_{i}=\sum_{j=1}^{n} \frac{\omega_{j}\left(z_{j}^{+}-z_{i j}\right)}{z_{j}^{+}-z_{j}^{-}},
$$

$$
R_{i}=\max _{j} \frac{\omega_{j}\left(z_{j}^{+}-z_{i j}\right)}{z_{j}^{+}-z_{j}^{-}} .
$$

The value of $S_{i}$ is the group benefit value, which is to ensure that all the indicators can be considered in the decision-making. The $R_{i}$ is the largest individual regret value, which is to consider the impact of the largest deviation index on the scheme ranking.

Direct calculations of Pythagorean fuzzy numbers lose too much important raw information. We use the Hamming distance.

$$
\begin{aligned}
d\left(z_{1}, z_{2}\right)= & \frac{1}{4}\left(\left|\left(\mu_{z_{1}}^{L}\right)^{p}-\left(\mu_{z_{2}}^{L}\right)^{p}\right|+\left|\left(\mu_{z_{1}}^{U}\right)^{p}-\left(\mu_{z_{2}}^{U}\right)^{p}\right|\right. \\
& +\left|\left(v_{z_{1}}^{L}\right)^{p}-\left(v_{z_{2}}^{L}\right)^{p}\right|+\left|\left(v_{z_{1}}^{U}\right)^{p}-\left(v_{z_{2}}^{U}\right)^{p}\right| \\
& +\left|\left(\pi_{z_{1}}^{L}\right)^{p}-\left(\pi_{z_{2}}^{L}\right)^{p}\right|+\left|\left(\pi_{z_{1}}^{U}\right)^{p}-\left(\pi_{z_{2}}^{U}\right)^{p}\right| .
\end{aligned}
$$

Among them, $z_{1}$ and $z_{2}$ are two interval-valued Pythagorean fuzzy numbers.

The improved $S_{i}$ and $R_{i}$ formulas are as follows.

$$
\begin{array}{r}
S_{i}=\sum_{j=1}^{n} \frac{d\left(z_{j}^{+}, z_{i j}\right)}{d\left(z_{j}^{+}, z_{j}^{-}\right)}, \\
R_{i}=\max _{j} \frac{d\left(z_{j}^{+}, z_{i j}\right)}{d\left(z_{j}^{+}, z_{j}^{-}\right)} .
\end{array}
$$




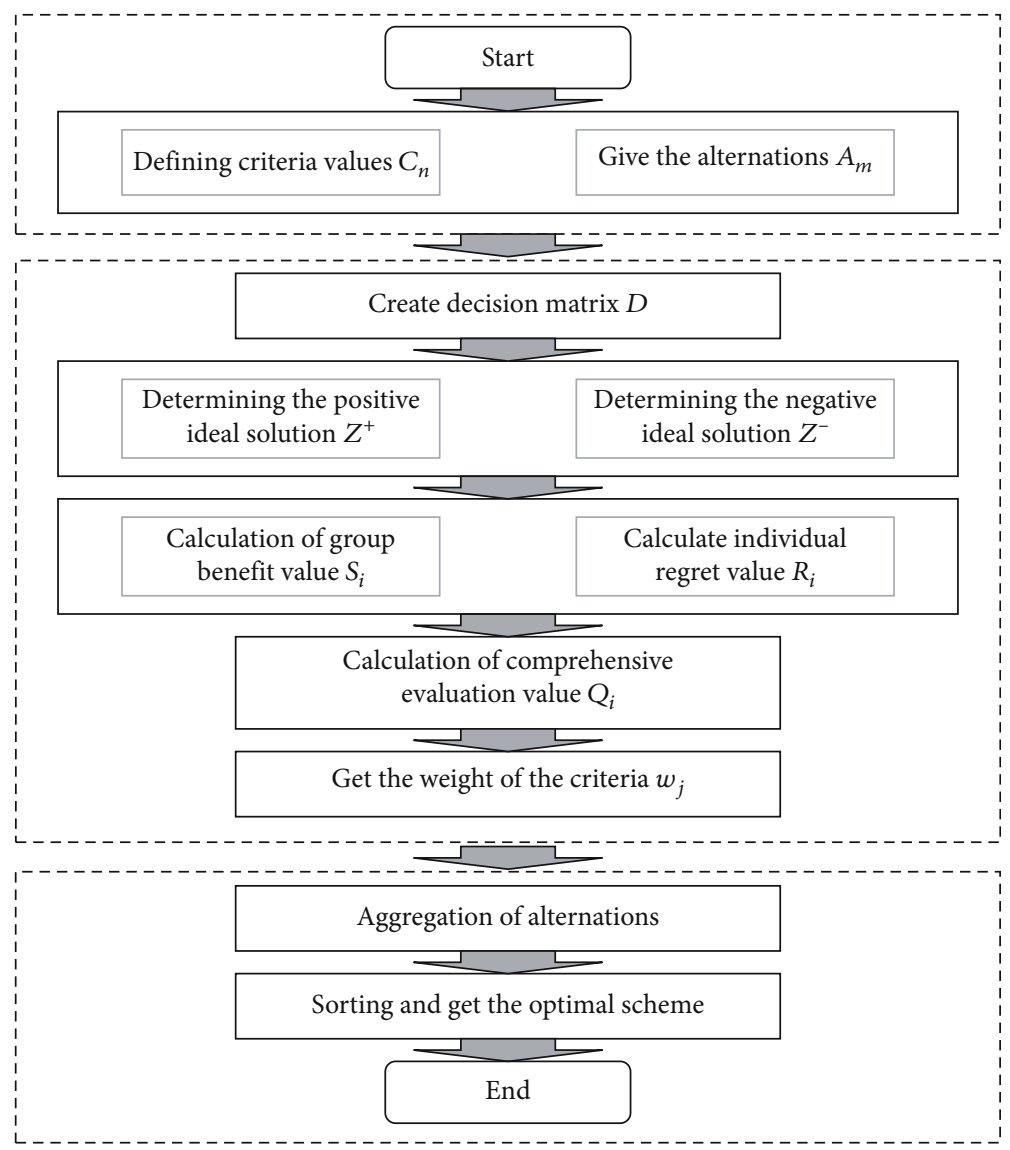

FIGURE 3: Working process of the proposed method.

To be able to take into account the value of group benefit and not ignore the maximum individual regret value, the following comprehensive evaluation methods for $S_{i}$ and $R_{i}$ are defined as follows.

$$
Q_{i}=v \frac{S_{i}-S^{-}}{S^{+}-S^{-}}+(1-v) \frac{R_{i}-R^{-}}{R^{+}-R^{-}}
$$

where $v$ is the adjustment parameter of decision mechanism. When $v>0.5$, the decision to maximize the group benefit is adopted; when $v<0.5$, the decision to maximize the maximum regret of the individual is adopted, and when $v=0.5$, the compromise decision strategy is adopted, which not only pays attention to the overall situation of the scheme but also takes into account the situation of a single multi-index. By doing this, it pursues the maximization of comprehensive benefits and the minimization of individual losses. In addition, $S^{+}=\max _{i} S_{i}, S^{-}=\min _{i} S_{i}, R^{+}=\max _{i} R_{i}$, and $R^{-}=\min _{i} R_{i}$.

4.2.3. Weight Determination Method. Based on the consideration of practical problems and the relevance of practical problems, we adopt the method of integrating subjective and objective weight, set up a regulating parameter $\lambda(0<\lambda$ $<1$ ), and calculate the final weight of each evaluation criteria. The subjective weight given by each evaluation criteria is assumed to be $\omega_{i}^{s}=\left(\omega_{1}^{s}, \omega_{2}^{s}, \cdots, \omega_{n}^{s}\right)$, while the objective weight $\omega_{i}^{o}$ is based on data and calculated on the basis of comprehensive evaluation values as follows.

$$
\omega_{i}^{o}=\frac{Q i}{\sum_{i=1}^{n} Q i}
$$

where $\mathrm{Q}_{i}$ is a comprehensive evaluation and can be taken to calculate the objective weight. So it can reflect real data information.

Which,

$$
\omega_{i}^{o}=\left(\omega_{1}^{o}, \omega_{2}^{o}, \cdots, \omega_{n}^{o}\right)
$$

Integrated,

$$
\omega_{i}=\lambda \cdot \omega_{i}^{0}+(1-\lambda) \cdot \omega_{i}^{s} .
$$

The $\lambda$ is the adjustment parameter of subjective and objective weight. When $>0.5$, the objective weight is larger and more attention to objective data; when $\lambda<0.5$, the subjective weight is larger and more attention to expert judgment. As $\lambda=0.5$, the subjective and objective weight is equally important. $\omega_{i}$ is the final weight of each alternative criteria.

4.3. Multicriteria Decision-Making Method under IntervalValued Pythagorean Fuzzy Environment. This section extends VIKOR method and aggregation operator to propose a new multicriteria interval-valued Pythagorean fuzzy decision method to solve practical problems. The proposed solution is shown in Figure 3. 


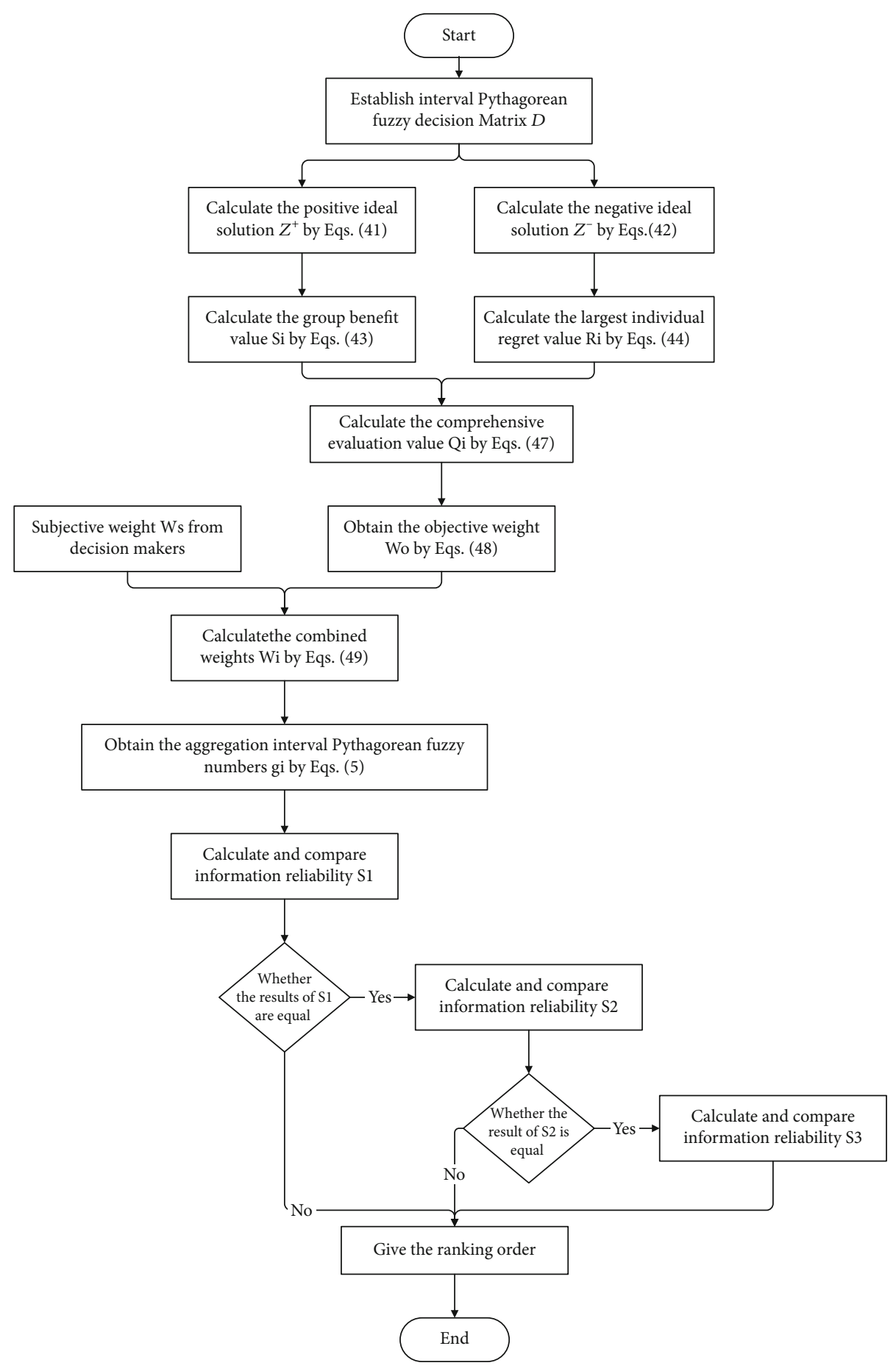

Figure 4: Flow chart of decision process.

A multicriteria decision-making method with intervalvalued Pythagorean fuzzy information may be summarized as follows.

Step 1. Establish interval-valued Pythagorean fuzzy decision matrix $D\left(A_{i} * C_{j}\right)$.

Step 2. Calculate the positive ideal solution $Z^{+}$by Eq. (47) and negative ideal solution $Z^{-}$by Eq. (48).
Step 3. Calculate the group benefit value $S_{i}$ by Eq. (49) and the largest individual regret value $R_{i}$ by Eq. (50) and make use of $S_{i}$ and $R_{i}$ to calculate the comprehensive evaluation value $Q_{i}$ by Eq. (54).

Step 4. Obtain the objective weight $\omega_{i}^{o}$ by Eq. (55) and then combine it with the subjective weight $\omega_{i}^{s}$ from expert to get the combined weights $\omega_{i}$ by Eq. (57). 
TABle 2: Decision matrix.

\begin{tabular}{llccc}
\hline & C1 & C2 & C3 & C4 \\
\hline A1 & $([0.5,0.6],[0.2,0.3])$ & $([0.6,0.7],[0.3,0.4])$ & $([0.5,0.8],[0.5,0.6])$ & $([0.3,0.6],[0.3,0.4])$ \\
A2 & $([0.4,0.8],[0.3,0.4])$ & $([0.7,0.9],[0.2,0.3])$ & $([0.6,0.8],[0.3,0.4])$ & $([0.6,0.7],[0.2,0.3])$ \\
A3 & $([0.5,0.6],[0.1,0.3])$ & $([0.6,0.7],[0.2,0.4])$ & $([0.5,0.8],[0.4,0.6])$ & $([0.3,0.6],[0.2,0.4])$ \\
A4 & $([0.3,0.7],[0.3,0.4])$ & $([0.6,0.8],[0.2,0.3])$ & $([0.5,0.7],[0.3,0.4])$ & $([0.5,0.6],[0.2,0.3])$ \\
\hline
\end{tabular}

Step 5. Obtain the aggregation interval-valued Pythagorean fuzzy numbers $g_{j}$ by Eq. (5).

Step 6. Calculate the information reliability $S_{1}, S_{2}$, and $S_{3}$ (Eqs. (37)-(39)) of alternatives $A_{i}$ by using the interval-valued Pythagorean fuzzy number set $g_{j}$.

Step 7. The ranking method proposed in this paper is aimed at calculating the score function by Eq. (40) of each scheme, and the better scheme is obtained by ranking rules.

The flow chart of decision process is shown in Figure 4.

\section{Application Example}

In order to illustrate the feasibility of the proposed method in solving the multicriteria decision problem, this paper takes the construction of wireless communications infrastructure as an example to illustrate the decision analysis process and makes a comparison of this method with other methods.

Due to the rapid development of the Internet and the continuous upgrading of communication technology, we are ushered to the era of high-speed mobile communication network. The development of 5G technology can deal with the growth of more traffic mobile services in the future and massive new services as well as new devices. 5G technology has been studied further. The launch of $5 \mathrm{G}$ of mobile communication technology standard industry in 2016 accelerated the pace of $5 \mathrm{G}$ technology research and development. China regards information technology as the primary task of science and technology development and thus continuously increases 5G R\&D investment. 5G technology satisfies the Internet and people's demand for communication stability. It has been officially commercialized. People enjoy the efficient and convenient life brought by high-speed network. When mobile phones are used to watch TV, video call, watch live broadcast, and on-line courses and do other activities, the efficiency of network transmission and the fluency of viewing has been significantly improved, which has brought better experience for users.

However, with the development of communication network and communication service, there are many new problems in the construction and protection of communication infrastructure. It is also difficult to choose the base station. The site selection unit needs to make a comprehensive programmer on the siting of base station and other relevant parameters on the basis of the environmental and business distribution characteristics of the covered area so as to balance the coverage, capacity, cost, and quality of wireless net-
TABLE 3: Distance matrix from the positive solution.

\begin{tabular}{lcccc}
\hline & C1 & C2 & C3 & C4 \\
\hline A1 & 0.0941 & 0.1127 & 0.0834 & 0.0947 \\
A2 & 0.0405 & 0.0000 & 0.0000 & 0.0000 \\
A3 & 0.0990 & 0.1199 & 0.0661 & 0.0973 \\
A4 & 0.0673 & 0.0757 & 0.0658 & 0.0602 \\
\hline
\end{tabular}

work in this area. $5 \mathrm{~g}$ development, base station first. 5G base station is not only an important part of the new infrastructure construction but also the foundation of $5 \mathrm{~g}$ technology to bring economic output. Therefore, it is urgent to solve this problem. The siting of $5 \mathrm{G}$ base station should be based on the total cost of its construction, the cost of signal coverage, system capacity, and network communication quality. Evaluation criteria should be established as $C_{j}(j=1,2,3,4)$. The experts have proposed four plans $A_{i}$ $(i=1,2,3,4)$. Based on previous experience, network operators evaluate each scheme and make decisions matrix as shown in Table 2. Experts indicated that four programmers have its own strengths. And the corresponding weight is $\omega_{i}^{s}=\{0.25 ; 0.25 ; 0.25 ; 0.25\}$.

According to the expert evaluation and weight information, the expert calculates the scores of the four schemes and obtains the better scheme.

On the basis of the decision matrix, the positive ideal solution group of the criteria $Z_{j}^{+}(j=1,2,3,4)$ and the negative ideal solution $Z_{j}^{-}(j=1,2,3,4)$ are calculated as follows.

$$
\begin{aligned}
& Z_{j}^{+}=\{([0.5,0.8],[0.1,0.3]),([0.7,0.9],[0.2,0.3]), \\
&([0.6,0.8],[0.3,0.4]),([0.6,0.7],[0.2,0.3])\}, \\
& Z_{j}^{-}=\{([0.3,0.6],[0.3,0.4]),([0.6,0.7],[0.3,0.4]),([0.5,0.7],[0.5,0.6]),([0.3,0.6],[0.3,0.4])\} .
\end{aligned}
$$

According to formula (50), the distance between each evaluation value and positive ideal solution is calculated as the matrix composed of $d\left(z_{j}^{+}, z_{i j}\right)$ shown in Table 3 , and the distance between the positive and negative ideal solutions is obtained from the vector $D=[0.1011,0.1127,0.0823,0.0947]$.

According to formula (52), the group benefit value is calculated as $S_{i}(i=1,2,3,4)$ 


$$
\begin{gathered}
S_{1}=\sum_{j=1}^{n} \frac{d\left(z_{j}^{+}, z_{i j}\right)}{d\left(z_{j}^{+}, z_{j}^{-}\right)}=\frac{0.0941}{0.1011}+\frac{0.0405}{0.1011}+\frac{0.0990}{0.1011}+\frac{0.0673}{0.1011}=2.9772, \\
S_{2}=\frac{0.1127}{0.1127}+\frac{0.0000}{0.1127}+\frac{0.1199}{0.1127}+\frac{0.0757}{0.1127}=2.7358, \\
S_{3}=\frac{0.0834}{0.0823}+\frac{0.0000}{0.0823}+\frac{0.0661}{0.0823}+\frac{0.0658}{0.0823}=2.6163, \\
S_{4}=\frac{0.0947}{0.0947}+\frac{0.0000}{0.0947}+\frac{0.0973}{0.0947}+\frac{0.0602}{0.0947}=2.6630 .
\end{gathered}
$$

According to formula (53), the matrix of individual regret value $R_{i}(i=1,2,3,4)$ is showed in Table 4 . 1.0275

Got that $R_{1}=0.9796, R_{2}=1.0644, R_{3}=1.0137$, and $R_{4}=$

According to formula (54), the comprehensive evaluation value of each criteria is calculated. The value of $v$ here is $v$ $=0.5$, which indicates that a compromise decision strategy is adopted, and the overall situation of the scheme and the case of a single multi-index are taken into account. The distance formula in the example adopts Euclidean distance.

$$
\begin{gathered}
Q_{1}=0.5 * \frac{2.9772-2.6163}{2.9772-2.6163}+(1-0.5) * \frac{0.9796-0.9796}{1.0664-0.9796}=0.5000, \\
Q_{2}=0.5 * \frac{2.7358-2.6163}{2.9772-2.6163}+(1-0.5) * \frac{1.0664-0.9796}{1.0664-0.9796}=0.6655, \\
Q_{3}=0.5 * \frac{2.6163-2.6163}{2.9772-2.6163}+(1-0.5) * \frac{1.0137-0.9796}{1.0664-0.9796}=0.2014, \\
Q_{4}=0.5 * \frac{2.6630-2.6163}{2.9772-2.6163}+(1-0.5) * \frac{1.0275-0.9796}{1.0664-0.9796}=0.3471, \\
Q_{i}=\{0.5000 ; 0.6655 ; 0.2014 ; 0.3471\},(i=1,2,3,4) .
\end{gathered}
$$

According to formula (55) and the comprehensive evaluation value, the objective weight of the alternative scheme is calculated.

$$
\begin{gathered}
\omega_{1}^{o}=\frac{0.5000}{0.5000+0.6655+0.2014+0.3471}=0.2917, \\
\omega_{2}^{o}=\frac{0.6655}{0.5000+0.6655+0.2014+0.3471}=0.3883, \\
\omega_{3}^{o}=\frac{0.2014}{0.5000+0.6655+0.2014+0.3471}=0.1175, \\
\omega_{4}^{o}=\frac{0.3471}{0.5000+0.6655+0.2014+0.3471}=0.2025, \\
\omega_{i}^{o}=\{0.2917 ; 0.3883 ; 0.1175 ; 0.2025\},(i=1,2,3,4) .
\end{gathered}
$$

According to formula (57), the subjective and objective weight is considered synthetically. As for this case, the subjective and objective weight is equally important. The $\lambda=$ 0.5 , this means the adoption of a more compromise value combination.
TABLE 4: Total regret matrix.

\begin{tabular}{ccccc}
\hline & $\mathrm{C} 1$ & $\mathrm{C} 2$ & $\mathrm{C} 3$ & $\mathrm{C} 4$ \\
\hline $\mathrm{A} 1$ & 0.9316 & 1.0000 & 1.0137 & 1.0000 \\
$\mathrm{~A} 2$ & 0.4004 & 0.0000 & 0.0000 & 0.0000 \\
$\mathrm{~A} 3$ & 0.9796 & 1.0644 & 0.8036 & 1.0275 \\
$\mathrm{~A} 4$ & 0.6656 & 0.6714 & 0.799 & 0.6355 \\
\hline
\end{tabular}

$$
\begin{aligned}
\omega_{i}= & \lambda \cdot \omega_{i}^{0}+(1-\lambda) \cdot \omega_{i}^{s}=0.5 *\{0.2917 ; 0.3883 ; 0.1175 ; 0.2025\} \\
& +(1-0.5) *\{0.25 ; 0.25 ; 0.25 ; 0.25\} \\
= & \{0.2709 ; 0.3191 ; 0.1838 ; 0.2263\},(i=1,2,3,4) .
\end{aligned}
$$

Therefore, we can calculate that the combined weights of the four options are $\omega_{1}=0.2709, \omega_{2}=0.3191, \omega_{3}=0.1838$, and $\omega_{4}=0.2263$.

By formula (5), each scheme is assembled.

$$
\begin{aligned}
& g_{1}=\left\langle\left[1-(1-0.5)^{0.3208} \prod_{j=1}^{m}\left(1-\mu_{j L}\right)^{\omega^{j}}, 1-\prod_{j=1}^{m}\left(1-\mu_{j U}\right)^{\omega^{j}}\right],\right. \\
&\left.\cdot\left[\prod_{j=1}^{m} v_{j L} \omega^{j}, \prod_{j=1}^{m} v_{j U} \omega^{j}\right]\right\rangle, \\
& g_{1}=\langle[0.4975,0.6787],[0.2953,0.3986]\rangle, \\
& g_{2}=\langle[0.5927,0.8243],[0.2405,0.3419]\rangle, \\
& g_{3}=\langle[0.4975,0.6787],[0.1883,0.3986]\rangle, \\
& g_{4}=\langle[0.4899,0.7187],[0.2405,0.3419]\rangle .
\end{aligned}
$$

Finally, we calculate and analyze the score function of Pythagorean fuzzy number in the above four assembly intervals. According to formula (37), it can be calculated as follows.

$$
\begin{aligned}
& S_{1\left(g_{1}\right)}=\frac{1}{2} \pi_{p_{4}}^{2}=\frac{1}{2} *\left(\sqrt{1-0.6787^{2}-0.3986^{2}}\right)^{2}=0.3084 \\
& S_{1\left(g_{2}\right)}=\frac{1}{2} \pi_{p_{4}}^{2}=\frac{1}{2} *\left(\sqrt{1-0.8243^{2}-0.3419^{2}}\right)^{2}=0.2256 \\
& S_{1\left(g_{3}\right)}=\frac{1}{2} \pi_{p_{4}}^{2}=\frac{1}{2} *\left(\sqrt{1-0.6787^{2}-0.3986^{2}}\right)^{2}=0.3084 \\
& S_{1\left(g_{4}\right)}=\frac{1}{2} \pi_{p_{4}}^{2}=\frac{1}{2} *\left(\sqrt{1-0.7187^{2}-0.3419^{2}}\right)^{2}=0.3027 .
\end{aligned}
$$

According to the rules of judgment, the smaller $S_{1}$ is, the better the scheme. We conclude that $2>4>1 \sim 3$. We can see that the interval-valued Pythagorean fuzzy number $g_{1}([0.4975,0.6787],[0.2953,0.3986])$ and $g_{2}([0.5927,0.8243]$, $[0.2405,0.3419])$ is different. Based on the above ranking rule, when $S_{1\left(g_{1}\right)}=S_{1\left(g_{3}\right)}$, comparative $S_{2\left(g_{1}\right)}$ and $S_{2\left(g_{3}\right)}$. Utilization $S_{2}=(1 / 2) \pi_{p_{1}}^{2}-(1 / 2) \pi_{p_{3}}^{2}(37)$. 
TABLE 5: Results from various ranking methods.

\begin{tabular}{|c|c|c|c|c|}
\hline Method used & Score function & Alternative & Score value & $\begin{array}{l}\text { Ranking order of } \\
\text { alternatives }\end{array}$ \\
\hline \multirow{4}{*}{ Zhang [4] } & \multirow{4}{*}{$\zeta(\beta)=\frac{2-\left(v_{\beta}^{L}\right)^{2}-\left(v_{\beta}^{U}\right)^{2}}{4-\left(\mu_{\beta}^{L}\right)^{2}-\left(\mu_{\beta}^{U}\right)^{2}-\left(v_{\beta}^{L}\right)^{2}-\left(v_{\beta}^{U}\right)^{2}}$} & $\mathrm{~A} 1$ & 0.5759 & \multirow{4}{*}{$2>4>3>1$} \\
\hline & & A2 & 0.6532 & \\
\hline & & A3 & 0.5830 & \\
\hline & & A4 & 0.5948 & \\
\hline \multirow{4}{*}{ Garg [5] } & $S(P)=\frac{a^{2}+b^{2}-c^{2}-d^{2}}{2}$ & $\mathrm{~A} 1$ & 0.4771 & \multirow{4}{*}{$2>1>4>3$} \\
\hline & $H(P)=\frac{a^{2}+b^{2}+c^{2}+d^{2}}{2}$ & A2 & 0.6027 & \\
\hline & $M(P)=\frac{a^{2}-\sqrt{1-a^{2}-c^{2}}+b^{2}-\sqrt{1-b^{2}-d^{2}}}{}$ & A3 & 0.4512 & \\
\hline & $M_{g}(P)=\frac{2}{2}$ & $\mathrm{~A} 4$ & 0.4656 & \\
\hline \multirow{4}{*}{ Garg [48] } & & A1 & 0.9723 & \multirow{4}{*}{$2>4>3>1$} \\
\hline & $\quad(P)=a^{2}+b^{2} \sqrt{1-a^{2}-c^{2}}+b^{2}+a^{2} \sqrt{1-b^{2}-d^{2}}$ & A2 & 1.3712 & \\
\hline & $M_{\text {Grag }}(P)=$ & A3 & 0.9795 & \\
\hline & & A4 & 1.04560 & \\
\hline \multirow{4}{*}{ Grag [46] } & \multirow{4}{*}{$M(P)=\frac{\left(a^{2}-c^{2}\right)\left(1+\sqrt{1-a^{2}-c^{2}}\right)+\left(b^{2}-d^{2}\right)\left(1+\sqrt{1-b^{2}-d^{2}}\right)}{2}$} & A1 & 0.3895 & \multirow{4}{*}{$2>4>3>1$} \\
\hline & & A2 & 0.6677 & \\
\hline & & A3 & 0.4397 & \\
\hline & & A4 & 0.4882 & \\
\hline \multirow{4}{*}{ Нo [47] } & \multirow{4}{*}{$\mathrm{CI}(P)=\eta \cdot \mathrm{CC}_{a}^{w}\left(P_{i}\right)+(1-\eta) \cdot \mathrm{CC}_{v}^{w}\left(P_{i}\right)$} & A1 & 0.4830 & \multirow{4}{*}{$4>3>2>1$} \\
\hline & & A2 & 0.4850 & \\
\hline & & A3 & 0.4923 & \\
\hline & & A4 & 0.4992 & \\
\hline \multirow{5}{*}{$\begin{array}{l}\text { This paper } \\
\text { proposed }\end{array}$} & & & S1 & \multirow{5}{*}{$2>4>3>1$} \\
\hline & $S 1=\frac{1}{2} \pi_{p_{4}}^{2}$ & Al & $0.3084 \quad 0.0716$ & \\
\hline & $S_{2}=\frac{1}{2} \pi_{p_{1}}^{2}-\frac{1}{2} \pi_{p_{3}}^{2}$ & A2 & $0.2256 \quad-$ & \\
\hline & $S_{3}=\frac{1}{2} \pi_{p_{1}}^{2}-\frac{1}{2} \pi_{p_{2}}^{2}$ & $\mathrm{~A} 3$ & $0.3084 \quad 0.0685$ & \\
\hline & & A4 & $0.3027 \quad-$ & \\
\hline
\end{tabular}

$S_{2\left(g_{1}\right)}=\frac{1}{2} *\left(\sqrt{1-0.4975^{2}-0.2953^{2}}\right)^{2}-\frac{1}{2} *\left(\sqrt{1-0.6787^{2}-0.2953^{2}}\right)^{2}=0.0716$,
$S_{2\left(g_{3}\right)}=\frac{1}{2} *\left(\sqrt{1-0.4975^{2}-0.1883^{2}}\right)^{2}-\frac{1}{2} *\left(\sqrt{1-0.6787^{2}-0.1883^{2}}\right)^{2}=0.0685$.

According to the judgment rule (40), the smaller the $S_{2}$, the better the scheme, and we conclude that $3>1$. To sum up, we can draw $2>4>3>1$.

\section{Comparative Analysis}

Various ranking methods are employed to rank the candidates in the selection of wireless communication base station, and the results are shown in Table 5.

If there is an interval-valued Pythagorean fuzzy number $p=([a, b],[c, d])$ and it satisfies the requirements of $c=b$ and $d^{2}=1-b^{2}$, then we can simplify the formulas (28)-(30) as follows:

$$
S(P)=\frac{a^{2}+b^{2}-c^{2}-d^{2}}{2}=\frac{a^{2}+b^{2}-1}{2},
$$

$$
\begin{gathered}
H(P)=\frac{a^{2}+b^{2}+c^{2}+d^{2}}{2}=\frac{a^{2}+b^{2}+1}{2}, \\
M_{g}(P)=\frac{a^{2}-\sqrt{1-a^{2}-c^{2}}+b^{2}-\sqrt{1-b^{2}-d^{2}}}{2} \\
=\frac{a^{2}+b^{2}-\sqrt{1-a^{2}+b^{2}}}{2} .
\end{gathered}
$$

There are two interval-valued Pythagorean fuzzy numbers, $p_{1}=\left(\left[a_{1}, b_{1}\right],\left[c_{1}, d_{1}\right]\right)$ and $p_{2}=\left(\left[a_{2}, b_{2}\right],\left[c_{2}, d_{2}\right]\right)$. Suppose that $a_{1}+b_{1}=a_{2}+b_{2}=X$, then there always $S\left(p_{1}\right)=$ $S\left(p_{2}\right)=(X-1) / 2, H\left(p_{1}\right)=H\left(p_{2}\right)=(X+1) / 2$, and $M_{g}\left(p_{1}\right)$ $=M_{g}\left(p_{2}\right)=(X+\sqrt{1-X}) / 2$; therefore, we cannot judge the two interval-valued Pythagorean fuzzy numbers. There are some flaws in this method, so the result in method Garg [5] that $2>1>4>3$ is dubious. On the other hand, according to Table 2, the nonmembership of alternatives A2 and A4 are the same, and the membership of A2 is bigger than that of $\mathrm{A} 4$, so, $2>4$ is supposed to be the conclusion. But, the result in method Ho [47] shows us $4>3>2>1$; it is against the law $2>4$. So, the accuracy of method Ho [47] is also questionable. 


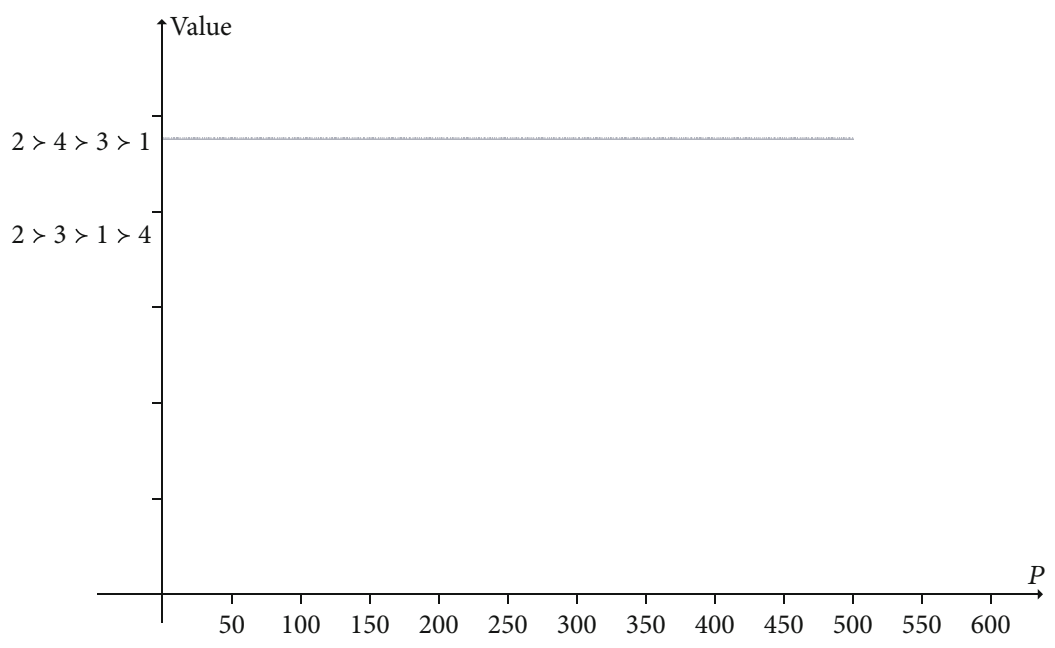

Figure 5: Influence of the change of $p$ value.

However, it is easy to be seen from the results in Table 5; the alternatives of numerical example show that methods Zhang [4], Garg [46], and Garg [48] are consistent with the conclusions of our method, i.e., $2 \succ 4>3>1$, and that is in line with logic. The results show that the proposed method is reliable. To prove this, a comparison between the ranking methods from previous studies and the ranking method proposed in this paper reveals two advantages of the latter: (1) the way of determining weight in decision-making process is more reasonable, and (2) the proposed method is more robust.

Ho [47] defines the parameter $\eta$ as the adjustment coefficient; it cannot adjust the subjective and objective weight of the decision data, but only determines the decision attitude of the whole scheme. However, when determining the objective weight, the method proposed in this paper based on the real data given by experts adopts the VIKOR method which considers the maximum group benefit and the minimum individual regret to obtain the comprehensive evaluation value of the alternative scheme. Then, the objective weight is determined, and the parameters are adjusted. The reference to historical experience is also important in solving practical problems because some experienced experts tend to value one or more evaluation criteria more. These more valuable criteria will be given greater weight. This paper combines the objective decision data given by experts, the experience of experts, the thoughts of supervisors, and the analysis of the specific problems so as to sort and select the alternatives more scientifically.

The distance formulas are used by the method in [47] and the proposed method in this paper to analyze and process the decision data. According to the Minkovsky distance formula used in this paper, the ranking results are very stable when the different values of $p$ are taken. For the commonly used distance formula such as Manhattan distance and Euclidean distance, this method is robust. To test the stability of the distance formula used in this paper, we have tested the robustness of the value of $p$ from 1 to 500. As shown in Figure 5, the experiment shows that when the value of $p$ is 2 to 500 ,

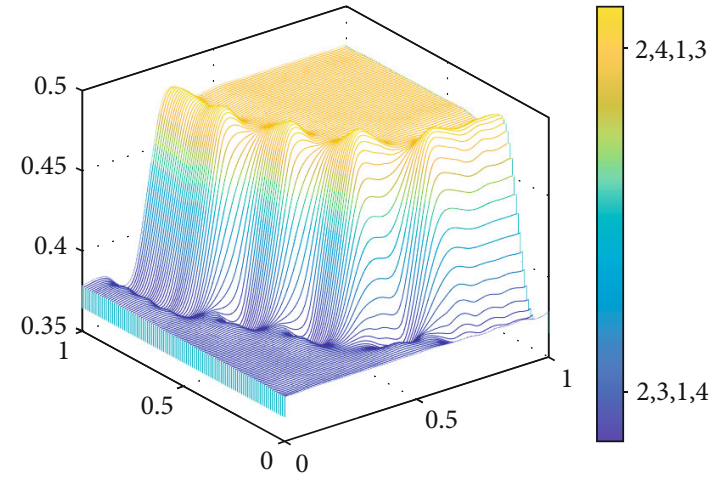

FIgURE 6: Waterfall of sensitivity analysis.

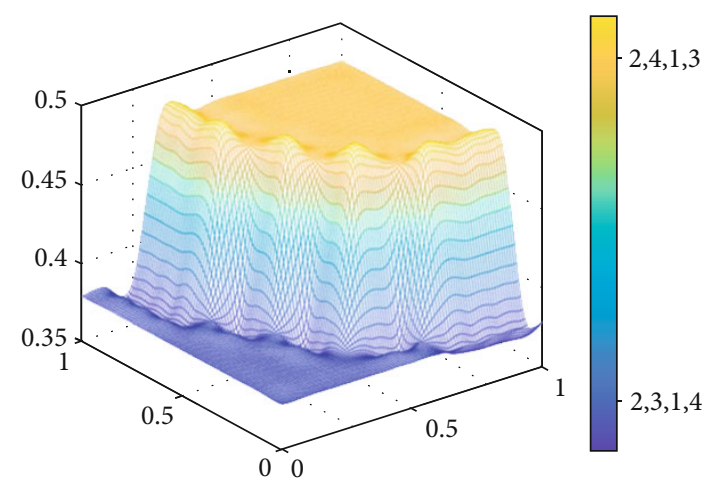

FIgURE 7: Contour lines of sensitivity analysis.

the ranking result is the same, and when the value of $p$ is 1 , the ranking result is not like the value we got previously, but, no matter what value the parameter $v$ and $\lambda$ is, it still gets the same result. So, the distance formula is very stable.

The ranking result of the alternative cannot be changed by the value of $p$. However, the distance formula used in [47] cannot obtain that result, so the method proposed by Ho [47] is robustness.

In the ranking method proposed in this paper, the interval-valued Pythagorean fuzzy number pair information 


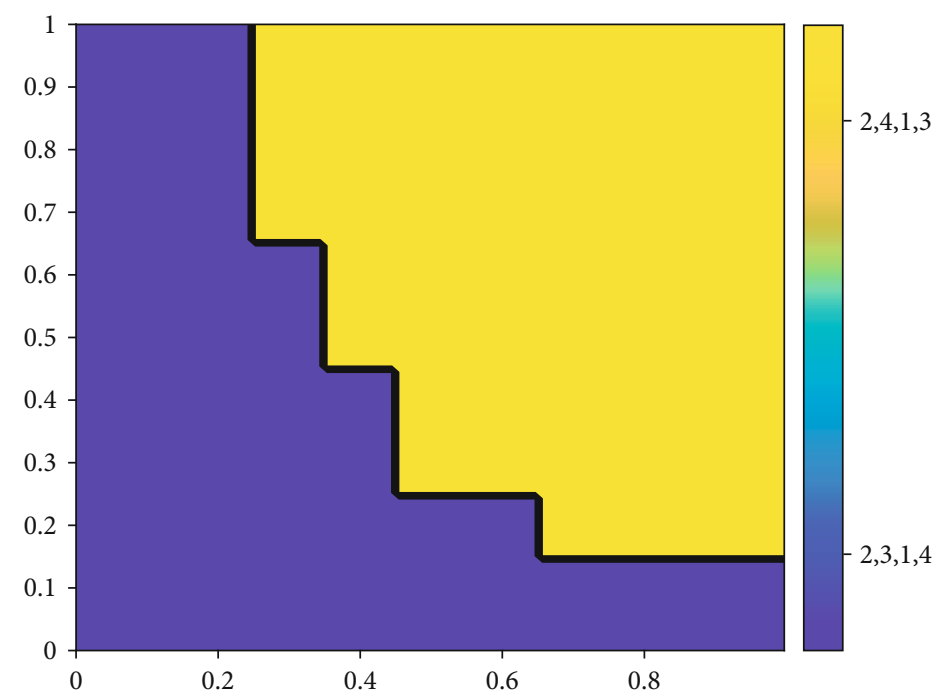

FIGURE 8: Thermal force diagram of sensitivity analysis.

aggregation is used in the last step, and the same type of interval-valued Pythagorean fuzzy number is finally obtained. The advantage of this method is that both the value range of membership degree and the value range of nonmembership degree are considered, which can effectively prevent the loss of decision information and minimize the impact of information aggregation on ranking results. The decision data in Table 1 clearly shows that Scheme 2 is obviously superior to Scheme 4, and the conclusion of the decision method proposed in [47]; the literature is $4>3>2$ $>1$, which is contrary to common sense judgment.

\section{Sensitivity Analysis}

A ranking method proposed in this paper sets the $v$ and $\lambda$ of adjusting parameters; parameter $v$ is used to regulate VIKOR relationship between maximizing group benefit and minimizing individual regret. Parameter $\lambda$ is used to adjust the relationship between objective weight and subjective weight. To achieve the convenience of studying the effect values of parameters $v$ and $\lambda$ on ranking results, we combine parameter $v$ and parameter $\lambda$ in the $[0,1]$ interval and observe their influence on the ranking results of alternatives. Experimental results show that with the different values of parameters $v$ and $\lambda$, the ranking results also change, and there are obvious laws.

In order to observe the influence of parameter changing on the ranking result of alternative scheme in a quick visual way, we use contour map, waterfall map, and thermodynamic map to express the ranking result.

As shown in Figures 6-8, the three graphs are contour plots, waterfall plots, and thermodynamic plots shown in the ranking results, respectively. It is easy to find that under the joint action of parameters $v$ and $\lambda$, there are two main distributions of the result value of the alternative, namely, result $1(2>4>3>1)$ and result $2(2>3>1>4)$. The observation shows that as the values of the parameters $v$ and $\lambda$ are close to 1 , the ranking is closer to the result 1 . When the values of parameters $v$ and $\lambda$ are close to 0 , the ranking is close to result
2. All the values of $v$ and $\lambda$ in the interval $[0,1]$ obey this law. Hence, we believe that this result is valid; the ranking method is stable, and the parameters have an effective moderating effect on the ranking results. With the uncertainty of data values in practical problems, the transition position of parameters and the law of variation of ranking results may be slightly different.

With regard to the ranking method proposed in this paper, parameter $v$ affects the weight of group benefit and individual regret preference, thus affecting the value of objective weight determined by VIKOR method. However, the parameter $\lambda$ affects the preference of subjective and objective, and the two parameters act together to adjust the decision scheme in different aspects. Experts can make more reasonable and meaningful decisions by analysing the actual problems and the needs of the actual situation and adopting different parameter combinations.

\section{Conclusions}

This paper overcomes some ranking problem that other interval-valued Pythagorean fuzzy sets cannot solve. As a whole, this study focuses on a new ranking function that combines traditional assembly operators with extended VIKOR functions to solve practical problems. The proposed method can effectively obtain a more feasible and practical result and improve the robustness of result. After comparing the results from previous studies with the one proposed in this paper, it can be concluded that the decision-making method in this paper is more stable. According to the previous discussion, some superiorities of the proposed approach are as follows.

(1) A new interval-valued Pythagorean fuzzy decision method not only expands the application scope of fuzzy decision but also adjusts the subjective and objective weight proportion precisely, which is important to solve practical problems 
(2) The ranking method proposed in this paper is very stable, and the ranking results will not change because of the change of the distance formula used. Therefore, the method is robust

(3) This paper stands on the shoulders of giants by inheriting and developing the study of previous research and apply the research result to practical usage

Although our proposed approach has played an important role in solving practical problems, this problem is still worthy of our further study. We look forward to proposing more practical decision methods and enriching fuzzy decision theory in the future. We will refer to the current research status of q-rung orthopair fuzzy decision-making and its application $[52,53]$ and consider expanding more ranking functions in q-rung orthopair fuzzy environment. Then, we integrate the extending function into the decision-making method of multidimensional preference linear programming to solve multicriteria decision-making problems. In the future, we will use this method to try to solve the problems of mobile transmission path selection and the selection of communication base station connection scheme in the field of wireless communication.

\section{Data Availability}

The example data used to support the findings of this study are available from the corresponding author upon request.

\section{Conflicts of Interest}

We declare that there is no conflict of interest regarding the publication of this paper.

\section{References}

[1] L. A. Zadeh, "Fuzzy sets," Information and Control, vol. 8, no. 3, pp. 338-353, 1965.

[2] K. T. Atanassov, "Intuitionistic fuzzy sets," Fuzzy Sets and Systems, vol. 20, no. 1, pp. 87-96, 1986.

[3] R. R. Yager, "Pythagorean membership grades in multicriteria decision making," IEEE Transactions on Fuzzy Systems, vol. 22, no. 4, pp. 958-965, 2014.

[4] X. Zhang, "Multicriteria Pythagorean fuzzy decision analysis: a hierarchical QUALIFLEX approach with the closeness indexbased ranking methods," Information Sciences, vol. 330, pp. 104-124, 2016.

[5] H. Garg, "A novel accuracy function under interval-valued Pythagorean fuzzy environment for solving multicriteria decision making problem," Journal of Intelligent \& Fuzzy Systems, vol. 31, no. 1, pp. 529-540, 2016.

[6] H. Garg, "A new improved score function of an interval-valued Pythagorean fuzzy set based TOPSIS method," International Journal for Uncertainty Quantification, vol. 7, no. 5, pp. 463474, 2017.

[7] T.-Y. Chen, "An interval-valued Pythagorean fuzzy outranking method with a closeness-based assignment model for multiple criteria decision making," International Journal of Intelligent Systems, vol. 33, no. 1, pp. 126-168, 2018.
[8] H. Garg, "New exponential operational laws and their aggregation operators for interval-valued Pythagorean fuzzy multicriteria decision-making," International Journal of Intelligent Systems, vol. 33, no. 3, pp. 653-683, 2018.

[9] H. Garg, "Linguistic interval-valued Pythagorean fuzzy sets and their application to multiple attribute group decisionmaking process," Cognitive Computation, vol. 12, no. 6, pp. 1313-1337, 2020.

[10] H. Garg, "Neutrality operations-based Pythagorean fuzzy aggregation operators and its applications to multiple attribute group decision-making process," Journal of Ambient Intelligence and Humanized Computing, vol. 11, no. 7, pp. 30213041, 2020.

[11] X. Zhang, "A novel approach based on similarity measure for Pythagorean fuzzy multiple criteria group decision making," International Journal of Intelligent Systems, vol. 31, no. 6, pp. 593-611, 2016.

[12] X. Zhang and Z. Xu, "Extension of TOPSIS to multiple criteria decision making with Pythagorean fuzzy sets," International Journal of Intelligent Systems, vol. 29, no. 12, pp. 1061-1078, 2014.

[13] P. Ren, Z. Xu, and X. Gou, "Pythagorean fuzzy TODIM approach to multi-criteria decision making," Applied Soft Computing, vol. 42, pp. 246-259, 2016.

[14] Z. Ma and Z. Xu, "Symmetric Pythagorean fuzzy weighted geometric/averaging operators and their application in multicriteria decision-making problems," International Journal of Intelligent Systems, vol. 31, no. 12, pp. 1198-1219, 2016.

[15] X. Peng and J. Dai, “Approaches to Pythagorean fuzzy stochastic multi-criteria decision making based on prospect theory and regret theory with new distance measure and score function," International Journal of Intelligent Systems, vol. 32, no. 11, pp. 1187-1214, 2017.

[16] X. Zhang, "Pythagorean fuzzy clustering analysis: a hierarchical clustering algorithm with the ratio index-based ranking methods," International Journal of Intelligent Systems, vol. 33, no. 9, pp. 1798-1822, 2018.

[17] C.-L. Hwang and K. Yoon, "Methods for multiple attribute decision making," in Lecture Notes in Economics and Mathematical Systems, vol 186, pp. 58-191, Springer, Berlin, Heidelberg, 1981.

[18] C.-T. Chen, "Extensions of the TOPSIS for group decisionmaking under fuzzy environment," Fuzzy Sets and Systems, vol. 114 , no. 1 , pp. 1-9, 2000.

[19] T. Chen and C. Tsao, "The interval-valued fuzzy TOPSIS method and experimental analysis," Fuzzy Sets and Systems, vol. 159, no. 11, pp. 1410-1428, 2008.

[20] M. F. Ak and M. Gul, "AHP-TOPSIS integration extended with Pythagorean fuzzy sets for information security risk analysis," Complex \& Intelligent Systems, vol. 5, no. 2, pp. 113-126, 2019.

[21] A. Calik, "A novel Pythagorean fuzzy AHP and fuzzy TOPSIS methodology for green supplier selection in the Industry 4.0 era," Soft Computing, vol. 25, no. 3, pp. 2253-2265, 2021.

[22] H. Garg and K. Kumar, "A novel exponential distance and its based TOPSIS method for interval-valued intuitionistic fuzzy sets using connection number of SPA theory," Artificial Intelligence Review, vol. 53, no. 1, pp. 595-624, 2020.

[23] H. Garg, A. Keikha, and H. Mishmast Nehi, "Multipleattribute decision-making problem using TOPSIS and Choquet integral with hesitant fuzzy number information," 
Mathematical Problems in Engineering, vol. 2020, Article ID 9874951, 12 pages, 2020.

[24] H. Garg and R. Arora, "TOPSIS method based on correlation coefficient for solving decision-making problems with intuitionistic fuzzy soft set information," AIMS Mathematics, vol. 5, no. 4, pp. 2944-2966, 2020.

[25] L. F. A. M. Gomes and M. M. P. P. Lima, "TODIM: basics and application to multicriteria ranking of projects with environmental impacts," Foundations of Computing and Decision Sciences, vol. 16, no. 4, pp. 113-127, 1992.

[26] C. Wei, Z. Ren, and R. M. Rodríguez, "A hesitant fuzzy linguistic TODIM method based on a score function," International Journal of Computational Intelligence Systems, vol. 8, no. 4, pp. 701-712, 2015.

[27] D. K. Sen, S. Datta, and S. S. Mahapatra, "Extension of TODIM for decision making in fuzzy environment: a case empirical research on selection of industrial robot," International Journal of Services and Operations Management, vol. 26, no. 2, pp. 238-276, 2017.

[28] A. Biswas and B. Sarkar, "Interval-valued Pythagorean fuzzy TODIM approach through point operator-based similarity measures for multicriteria group decision making," Kybernetes, vol. 48, no. 3, pp. 496-519, 2019.

[29] M. Gul, E. Celik, N. Aydin, A. Taskin Gumus, and A. F. Guneri, "A state of the art literature review of VIKOR and its fuzzy extensions on applications," Applied Soft Computing, vol. 46, pp. 60-89, 2016.

[30] P. Gupta, M. K. Mehlawat, and N. Grover, "Intuitionistic fuzzy multi-attribute group decision-making with an application to plant location selection based on a new extended VIKOR method," Information Sciences, vol. 370-371, pp. 184-203, 2016.

[31] A. Hafezalkotob and A. Hafezalkotob, "Interval target-based VIKOR method supported on interval distance and preference degree for machine selection," Engineering Applications of Artificial Intelligence, vol. 2017, no. 57, pp. 184-196, 2017.

[32] Z. Hao, Z. Xu, H. Zhao, and R. Zhang, "Novel intuitionistic fuzzy decision making models in the framework of decision field theory," Information Fusion, vol. 33, pp. 57-70, 2017.

[33] T.-Y. Chen, "Remoteness index-based Pythagorean fuzzy VIKOR methods with a generalized distance measure for multiple criteria decision analysis," Information Fusion, vol. 41, pp. 129-150, 2018.

[34] H. Garg, J. Gwak, T. Mahmood, and Z. Ali, "Power aggregation operators and VIKOR methods for complex q-rung orthopair fuzzy sets and their applications," Mathematics, vol. 8, no. 4, p. 538, 2020.

[35] T.-Y. Chen, "A novel risk evaluation method of technological innovation using an inferior ratio-based assignment model in the face of complex uncertainty," Expert Systems with Applications, vol. 95, pp. 333-350, 2018.

[36] T.-Y. Chen, "An outranking approach using a risk attitudinal assignment model involving Pythagorean fuzzy information and its application to financial decision making," Applied Soft Computing, vol. 71, pp. 460-487, 2018.

[37] D. N. le, G. Nhu Nguyen, H. Garg, Q. T. Huynh, T. Ngoc Bao, and N. Ngoc Tuan, "Optimizing bidders selection of multi-round procurement problem in software project management using parallel max-min ant system algorithm," Computers Materials \& Continua, vol. 66, no. 1, pp. 9931010, 2020.
[38] T.-Y. Chen, “An interval-valued Pythagorean fuzzy compromise approach with correlation- based closeness indices for multiple-criteria decision analysis of bridge construction methods," Complexity, vol. 2018, Article ID 6463039, 29 pages, 2018.

[39] T.-Y. Chen, "A novel VIKOR method with an application to multiple criteria decision analysis for hospital-based postacute care within a highly complex uncertain environment," Neural Computing \& Applications, vol. 31, no. 8, pp. 39693999, 2019.

[40] H. Garg, G. Shahzadi, and M. Akram, "Decision-making analysis based on Fermatean fuzzy Yager aggregation operators with application in COVID-19 testing facility," Mathematical Problems in Engineering, vol. 2020, Article ID 7279027, 16 pages, 2020.

[41] H. Garg, "Guest Editorial: Neutrosophic decision making and applications in knowledge management," CAAI Transactions on Intelligence Technology, vol. 5, no. 2, p. 67, 2020.

[42] S. K. Giri, T. Garai, H. Garg, and S. Islam, "Possibilistic mean of generalized non-linear intuitionistic fuzzy number to solve a price and quality dependent demand multi-item inventory model," Computational and Applied Mathematics, vol. 40, no. 4, pp. 1-24, 2021.

[43] X. U. Ze-shui, "Methods for aggregating interval-valued intuitionistic fuzzy information and their application to decision making," Control and Decision, vol. 2, pp. 215219, 2007.

[44] K. Rahman, "Approaches to some induced Einstein geometric aggregation operators based on interval-valued Pythagorean fuzzy numbers and their application," New Mathematics and Natural Computation, vol. 16, no. 2, pp. 211-230, 2020.

[45] S.-P. Wan, Z. Jin, F. Wang, and Z. Jin, "A new ranking method for Pythagorean fuzzy numbers," in 2017 12th International Conference on Intelligent Systems And Knowledge Engineering, pp. 1-6, Nanjing, China, November 2017.

[46] H. Garg, "A linear programming method based on an improved score function for interval-valued Pythagorean fuzzy numbers and its application to decision-making," International Journal of Uncertainty, Fuzziness and KnowledgeBased Systems, vol. 26, no. 1, pp. 67-80, 2018.

[47] L.-H. Ho, Y.-L. Lin, and T.-Y. Chen, "A Pearson-like correlation-based TOPSIS method with interval-valued Pythagorean fuzzy uncertainty and its application to multiple criteria decision analysis of stroke rehabilitation treatments," Neural Computing \& Applications, vol. 32, no. 12, pp. 8265$8295,2020$.

[48] H. Garg, "A novel improved accuracy function for interval valued Pythagorean fuzzy sets and its applications in the decision-making process," International Journal of Intelligent Systems, vol. 32, no. 12, pp. 1247-1260, 2017.

[49] T. Kumar, R. Bajaj, and M. D. Ansari, "On accuracy function and distance measures of interval-valued Pythagorean fuzzy sets with application to decision making," Scientia Iranica, vol. 27, no. 4, pp. 2127-2139, 2020.

[50] S.-P. Wan, Z. Jin, and J.-Y. Dong, “A new order relation for Pythagorean fuzzy numbers and application to multiattribute group decision making," Knowledge and Information Systems, vol. 62, no. 2, pp. 751-785, 2020.

[51] S. Opricovic, "Multicriteria optimization of civil engineering systems," Faculty of Civil Engineering, Belgrade, vol. 2, no. 1, pp. 5-21, 1998. 
[52] H. Garg, "CN-q-ROFS: connection number-basedq-rung orthopair fuzzy set and their application to decision-making process," International Journal of Intelligent Systems, vol. 36, no. 7, pp. 3106-3143, 2021.

[53] D. Rani and H. Garg, "Complex intuitionistic fuzzy preference relations and their applications in individual and group decision-making problems," International Journal of Intelligent Systems, vol. 36, no. 4, pp. 1800-1830, 2021. 